\title{
Randomised Controlled Trials of Alcohol-Based Surgical Site Skin Preparation for the Prevention of Surgical Site Infections: Systematic Review and Meta-Analysis
}

\author{
Trisha N. Peel *, Eliza Watson (D) and Sue J. Lee (D) \\ Department of Infectious Diseases, Alfred Hospital and Central Clinical School, Monash University, \\ 85 Commercial Road, Melbourne, VIC 3004, Australia; eliza.watson@monash.edu (E.W.); \\ sue.lee@monash.edu (S.J.L.) \\ * Correspondence: trisha.peel@monash.edu; Tel.: +61-(3)-90762000; Fax: +61-(3)-90762431
}

check for updates

Citation: Peel, T.N.; Watson, E.; Lee, S.J. Randomised Controlled Trials of Alcohol-Based Surgical Site Skin Preparation for the Prevention of Surgical Site Infections: Systematic Review and Meta-Analysis. J. Clin. Med. 2021, 10, 663. https://doi.org/ $10.3390 /$ jcm10040663

Academic Editor: Loredana Sarmati Received: 15 January 2021

Accepted: 4 February 2021

Published: 9 February 2021

Publisher's Note: MDPI stays neutral with regard to jurisdictional claims in published maps and institutional affiliations.

Copyright: (c) 2021 by the authors. Licensee MDPI, Basel, Switzerland. This article is an open access article distributed under the terms and conditions of the Creative Commons Attribution (CC BY) license (https:// creativecommons.org/licenses/by/ $4.0 /)$.
Abstract: (1) Background: Surgical site skin preparation is an important approach to prevent postoperative wound infections. International guidelines recommend that alcohol-based combinations be used, however, the optimal combination remains uncertain. This study compares the effectiveness of alcohol-based chlorhexidine and alcohol-based iodophor for surgical site skin preparation for prevention of surgical site infections (SSIs). (2) Methods: Randomised controlled trials comparing alcohol-based interventions for surgical site skin preparation were included. The proportion of SSIs was compared using risk ratios (RR) with 95\% confidence intervals (95\% CI). The meta-analysis was performed with a fixed effect model using Mantel-Haenszel methods. As an a priori subgroup analysis SSI risk was examined according to different surgical procedural groups. (3) Results: Thirteen studies were included ( $n=6023$ participants). The use of chlorhexidine-alcohol was associated with a reduction in risk of SSIs compared with iodophor-alcohol (RR 0.790; 95\% CI 0.669, 0.932). On sub-group analysis, chlorhexidine-alcohol was associated with a reduction in SSIs in caesarean surgery (RR 0.614; 95\% CI 0.453, 0.831) however, chlorhexidine-alcohol was associated with an increased risk of SSI in bone and joint surgery (RR 2.667; 95\% CI 1.051, 6.765). When excluding studies at high risk of bias on sensitivity analysis, this difference in alcohol-based combinations for bone and joint surgery was no longer observed (RR 2.636; 95\% CI 0.995, 6.983). (4) Conclusions: The use of chlorhexidine-alcohol skin preparations was associated with a reduced risk of SSI compared to iodophor-alcohol agents. However, the efficacy of alcohol-based preparation agents may differ according to the surgical procedure group. This difference must be interpreted with caution given the low number of studies and potential for bias, however, it warrants further investigation into the potential biological and clinical validity of these findings.

Keywords: surgical site skin preparation; surgical site infection; infection prevention; meta-analysis

\section{Introduction}

Over 300 million surgeries are performed annually worldwide based on a 2012 estimate [1]. Surgical site infections (SSIs) remain a major, costly complication of surgical procedures [2-4]. The patient's skin bacteria is the major source of infecting pathogens involved in SSIs and is the target of infection prevention strategies such as surgical site skin preparation [5-8]. The three main agents used are chlorhexidine gluconate, iodophors or alcohol. Despite longstanding use, the optimal preparation remains an issue of controversy [9]. It is recommended that alcohol-based products, combining chlorhexidine with alcohol or iodophors with alcohol, be used in preference to aqueous-based products, based on improved efficacy demonstrated in randomised controlled trials and meta-analyses [6-9]. These agents have different mechanisms and duration of activity. Alcohol and chlorhexidine gluconate disrupt the cell wall of microorganisms, whereas iodophors act upon intracellular proteins of microorganisms $[6,10]$. Alcohol has no residual activity, iodophors 
exhibit persistence of bacteriostatic activity when on the skin and chlorhexidine gluconate has excellent residual activity $[5,6,10]$.

In the guidelines for the prevention of SSIs published by The Centers for Disease Control and Prevention (CDC) [8] and the World Health Organization (WHO) [7,8], there was consensus that alcohol-based preparations were associated with reduced risk of SSI compared to aqueous-based solutions $[7,8]$. These guidelines, however, gave conflicting recommendations on the optimal agent to combine with alcohol. In the CDC guidelines, no recommendation for a specific alcohol-based product was made based on high-quality evidence from six randomised controlled trials [8]. In comparison, the WHO specified that chlorhexidine-alcohol preparation should be used, based on data from six randomised controlled trials of moderate quality [7]. The trials included a range of different procedure types and no sub-group analyses were performed to examine whether the efficacy of products differed between different surgery types.

The objective of this systematic review and meta-analysis was to determine the comparative effectiveness of alcohol-based chlorhexidine and alcohol-based iodophor as surgical site skin preparation agents to prevent SSIs based on data from randomized controlled trials. A secondary objective was to determine if the effectiveness of alcohol-based agents differs between different surgical procedure types.

\section{Methods}

This systematic review and meta-analysis was developed in keeping with the Preferred Reporting Items for Systematic Reviews and Meta-Analyses (PRISMA) Statement [11]. The intervention of interest was surgical site skin preparation with alcohol-based solutions or powders applied to the participant's skin at the site overlying the planned surgical incision. The solutions could be applied as a single step (e.g., combination preparations such as $2 \%$ chlorhexidine-gluconate in $70 \%$ ethanol) or as two sequential steps (e.g., $10 \%$ povidone solution followed by $70 \%$ alcohol solution). Classes and sub-classes of surgical site skin preparation included, but were not limited to: chlorhexidine gluconate, povidone, iodine, alcohol and ethanol.

Randomised controlled trials (RCTs) in human participants that compared at least two of the alcohol-based interventions for surgical site skin preparation were eligible for inclusion. Studies that compared aqueous-based solutions to other aqueous-based solutions or to alcohol-based solutions were excluded. Studies comparing other antisepsis techniques (e.g., preoperative showering/bathing, impregnated drapes) were not included. Non-English language publications were excluded.

The primary outcome of interest was surgical site infection (SSI), based on the Centers for Disease Control and Prevention/National Healthcare Safety Network (CDC/NHSN) Surveillance definitions for SSIs [5,8]. In the event the trial did not apply the CDC definition, the study definition was documented and mapped to the CDC definition, where possible. Secondary outcomes of interest included: adverse events including skin irritation or allergic reactions and health economic data, including direct hospital costs, societal and quality of life data.

The following electronic databases were searched: Medline (via PubMed), OVID EMBASE, CINAHL and the Cochrane Library Databases. The strategy for electronic databases search is outlined in Appendix A. There was no time limit on the studies included. In addition, the reference list for included studies were reviewed for literature saturation. Study authors were not contacted, and the Grey Literature was not included. Database searches were completed August 2019.

Two authors (TP and EW) independently screened all titles and abstracts of identified studies using the Covidence ${ }^{\circledR}$ (Veritas Health Innovation LTD, Melbourne, Australia) web-based platform. The full text was retrieved and reviewed for selected abstracts. Disagreement was resolved through consensus. Systematic reviews and meta-analyses were reviewed to identify additional randomised controlled trials. Data were extracted from the selected studies independently by two authors (TP and EW). Data extraction included 
the procedure type, number of participants, duration of follow up, the authors' definition of surgical site infection, number of patients experiencing a surgical site infection and the interventions being compared. Arm level data were extracted. Risk of bias was assessed using a domain-based evaluation by two authors (TP and EW) independently [12]. Domains examined included random sequence generation, allocation concealment, blinding, attrition, and reporting biases. Disagreement was resolved through discussion and consensus. These data were extracted into RevMan ${ }^{\circledR}$ (Review Manager [Computer program]. Version 5.3. Copenhagen: The Nordic Cochrane Centre, The Cochrane Collaboration, 2014). The protocol for the systematic review and meta-analysis was registered with PROSPERO (CRD42020148548).

The proportion of SSIs was compared between arms using risk ratios (RR) with 95\% confidence intervals $(95 \% \mathrm{CI})$. Statistical heterogeneity was quantified using the $\mathrm{I}^{2}$ statistic with a threshold at $\geq 50 \%$. A negative $\mathrm{I}^{2}$ statistic was regarded as no heterogeneity. Funnel plots were examined for asymmetry applying the approach outlined by Sterne et al. [13]. Disclosed conflicts of interest were also reviewed.

The meta-analysis was performed with a fixed effect model using Mantel-Haenszel methods to obtain the pooled relative risk (RR) estimate [14,15]. In the event significant heterogeneity $\left(\mathrm{I}^{2} \geq 50 \%\right)$ was observed, a random effects model using the restricted maximum likelihood method was performed. To account for trials with zero counts in either or both arms, a fixed continuity correction of 0.5 was added to each zero cell [16]. Sensitivity analyses were performed excluding trials with zero total events and, excluding trials with high risk of bias.

As part of the planned subgroup analysis the difference in risk of infection was examined according to different classifications of SSI (superficial and, deep or organ/space) and different surgical procedural groups. Data were analysed using STATA (16.0 College Station, TX, USA) with the META function.

\section{Results}

The initial search identified 4606 citations (Figure 1). Fifty-nine publications were subsequently retrieved for full-text review, of which 46 did not meet the inclusion criteria. Thirteen studies were eligible for inclusion in the review ( $n=6023$ participants) [17-29]. Characteristics of the included studies are outlined in Table 1. 


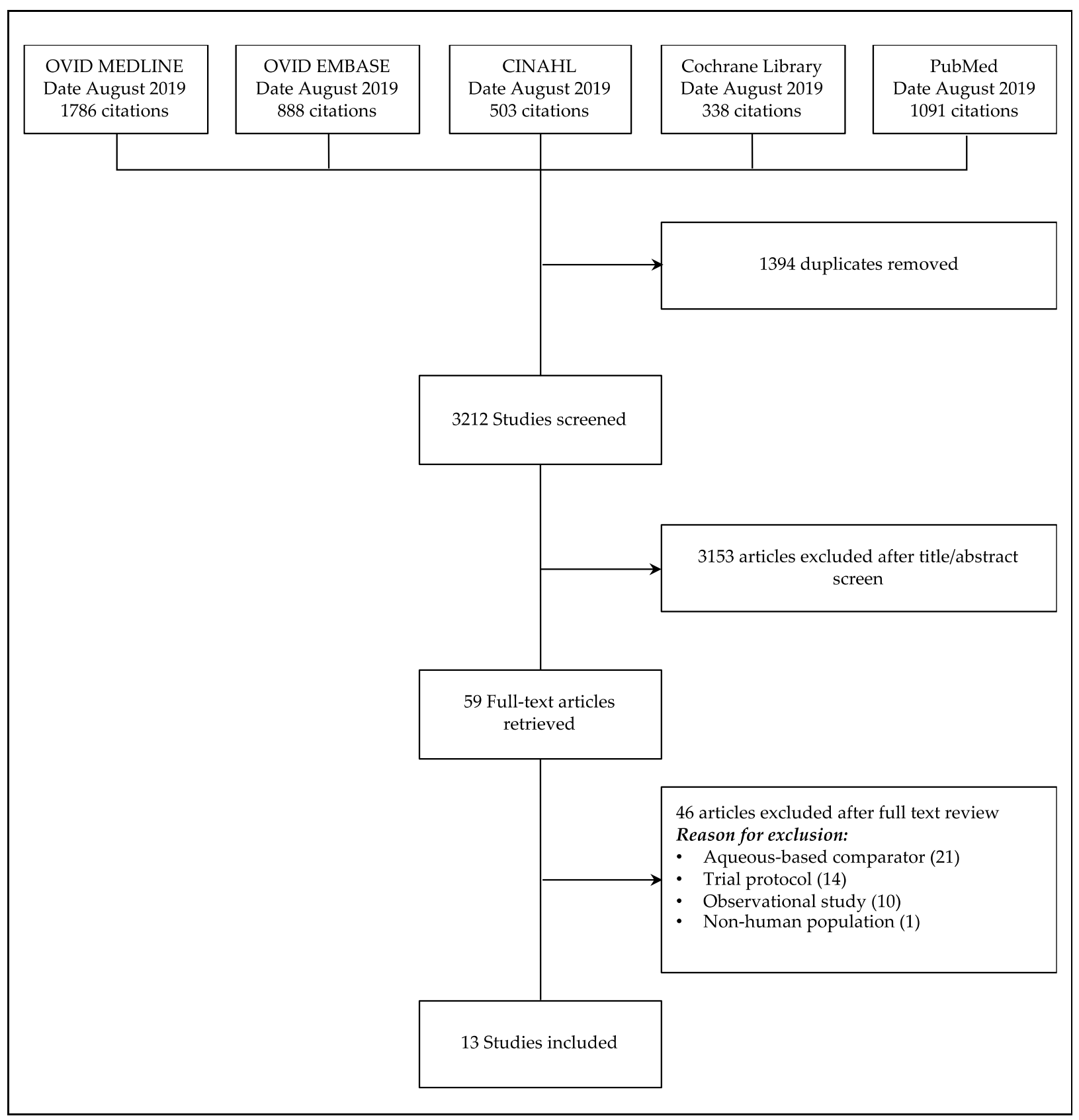

Figure 1. PRISMA Flow Chart of Systematic Review and Study Selection. 
Table 1. Characteristics of Included Studies.

\begin{tabular}{|c|c|c|c|c|c|c|}
\hline Study & Methods & Surgery Type & Interventions & Outcomes & Results & Notes \\
\hline $\begin{array}{l}\text { Berry } \\
1982 \\
{[17]}\end{array}$ & $\begin{array}{l}\text { Cluster randomised controlled } \\
\text { trial } \\
\text { Clustered by given day of } \\
\text { surgery } \\
\text { Single centre study }\end{array}$ & $\begin{array}{l}\text { Operations on biliary tract, } \\
\text { large bowel, laparotomy, } \\
\text { operations on hernia, } \\
\text { genitalia, varicose veins } \\
\text { and other 'clean' } \\
\text { non-abdominal operations }\end{array}$ & $\begin{array}{l}\text { Group A } \\
\text { Povidone-iodine } 10 \% \text { in alcohol } \\
\text { ( } n \text { total }=413 \text {; included in trial } \\
\text { analysis } n=413) \\
\text { Group B } \\
\text { Chlorhexidine ('Hibitane') } 0.5 \% \\
\text { in spirit } \\
\text { ( } n \text { total }=453 \text {; included in trial } \\
\text { analysis } n=453)\end{array}$ & $\begin{array}{l}\text { Wound infection defined as any } \\
\text { wound abnormality at time of } \\
\text { participant's discharge as } \\
\text { agreed by two observers. } \\
\text { Wounds were judged at each } \\
\text { inspection as fitting one or } \\
\text { more of the following } \\
\text { categories: normal, } \\
\text { erythematous, oedematous, } \\
\text { discharging or purulent. }\end{array}$ & $\begin{array}{l}\text { SSI } \\
\text { Iodophor-alcohol 61/413 (14.8\%) } \\
\text { Chlorhexidine-alcohol } 44 / 453(9.7 \%)\end{array}$ & $\begin{array}{l}\text { No sample size estimate provided } \\
\text { Follow up: until discharge } \\
\text { Data extracted from wound abnormalities at } \\
\text { discharge } \\
\text { (Table III in the publication by Berry et al.) } \\
\text { based on participants with "any abnormality" } \\
\text { No data provided on whether wound } \\
\text { abnormalities were superficial, deep or } \\
\text { organ/space }\end{array}$ \\
\hline $\begin{array}{l}\text { Broach } \\
2017 \\
{[18]}\end{array}$ & $\begin{array}{l}\text { Randomised controlled trial } \\
\text { Blinded } \\
\text { Non-inferiority trial design } \\
\text { Setting of study is not stated }\end{array}$ & $\begin{array}{l}\text { Elective } \\
\text { clean-contaminated } \\
\text { colorectal surgery }\end{array}$ & $\begin{array}{l}\text { Group A } \\
26 \mathrm{~mL} \text { single use applicator } \\
\text { containing iodine povacrylex } \\
{[0.7 \% \text { available iodine] } / 74 \%} \\
\text { isopropyl alcohol }(w / w) \\
{[“ \text { Duraprep" }]} \\
(n \text { total }=402 ; \text { included in trial } \\
\text { analysis } n=396) \\
\text { Group B } \\
26 \mathrm{~mL} \text { single-use applicator } \\
\text { containing } 2 \% \text { chlorhexidine } \\
\text { gluconate }(w / v) \text { and } 70 \% \\
\text { isopropyl alcohol }(v / v) \\
{[“ C h l o r a P r e p ”]} \\
(n \text { total }=400 ; \text { included in trial } \\
\text { analysis } n=392)\end{array}$ & $\begin{array}{l}\text { Surgical site infection at } \\
30 \text { days post discharge }( \pm 5 \\
\text { days) applying the CDC } \\
\text { definition of superficial or deep } \\
\text { surgical site infection. } \\
\text { Cellulitis and organ/space SSI } \\
\text { included as a secondary } \\
\text { outcome measure }\end{array}$ & $\begin{array}{l}\text { SSI } \\
\text { All SSI } \\
\text { Iodophor-alcohol 90/396 (22.7\%) } \\
\text { Chlorhexidine-alcohol } 82 / 392(20.9 \%) \\
\text { Superficial SSI } \\
\text { Iodophor-alcohol 46/396 (11.6\%) } \\
\text { Chlorhexidine-alcohol } 40 / 392(10.2 \%) \\
\text { Deep SSI } \\
\text { Iodophor-alcohol 28/396 (7.1\%) } \\
\text { Chlorhexidine-alcohol 22/392 (5.6\%) } \\
\text { Organ space SSI } \\
\text { Iodophor-alcohol 16/396 (4.0\%) } \\
\text { Chlorhexidine-alcohol } 20 / 392(5.1 \%) \\
\text { Cellulitis } \\
\text { Iodophor-alcohol 19/396 (4.8\%) } \\
\text { Chlorhexidine-alcohol 14/392 (3.6\%) } \\
\text { Adverse reactions } \\
\text { No adverse reactions (skin } \\
\text { irritation/allergy) reported in either arm }\end{array}$ & $\begin{array}{l}\text { Sample size estimate provided } \\
\text { For the analysis, organ space SSI included } \\
\text { with data from primary outcome (superficial } \\
\text { and deep SSI) } \\
\text { Follow-up: } 30 \text { days } \pm 5 \text { days }\end{array}$ \\
\hline
\end{tabular}


Table 1. Cont.

\begin{tabular}{|c|c|c|c|c|c|c|}
\hline Study & Methods & Surgery Type & Interventions & Outcomes & Results & Notes \\
\hline $\begin{array}{l}\text { Cheng } \\
2009 \\
{[19]}\end{array}$ & $\begin{array}{l}\text { Randomised controlled trial } \\
\text { Single centre study }\end{array}$ & $\begin{array}{l}\text { Foot surgery including: } \\
\text { metatarsal osteotomies for } \\
\text { correction of hallux valgus } \\
\text { deformity, removal of } \\
\text { osteophytes from the first } \\
\text { metatarsal and correction of } \\
\text { lesser toe deformities }\end{array}$ & $\begin{array}{l}\text { Group A } \\
\text { alcoholic betadine (Ecolab } \\
\text { Videne Alcoholic tincture, } \\
\text { povidine-iodine } 10 \% \text { w } w / w(1 \% \\
w / w \text { available iodine)) } \\
(n \text { total }=25 ; \text { included in trial } \\
\text { analysis } n=25) \\
\\
\text { Group B } \\
\text { alcoholic chlorhexidine (Ecolab } \\
\text { Hydrex, clear Chlorhexidine } \\
\text { gluconate } 0.5 \% \text { w } w / \text { in } 70 \% v / v) \\
(n \text { total }=25 ; \text { included in trial } \\
\text { analysis } n=25)\end{array}$ & $\begin{array}{l}\text { Number of bacterial colony } \\
\text { forming units pre- and } \\
\text { post-treatment with surgical } \\
\text { site skin preparation taken } \\
\text { from three sites (medial } \\
\text { hallucal nail fold, interdigital } \\
\text { web-spaces and the dorsal } \\
\text { aspect of the first metatarsal } \\
\text { phalangeal joint). } \\
\text { Postoperative infection rate } \\
\text { was reported as a secondary } \\
\text { outcome. No definition for } \\
\text { infection provided }\end{array}$ & $\begin{array}{l}\text { Positive cultures } \\
\text { Iodine-alcohol: positive cultures } \\
\text { prior to preparation } 62 \text {, after } 9 \text { from } \\
3 \text { sites in } 25 \text { participants } \\
\text { Chlorhexidine-alcohol: positive } \\
\text { cultures prior to preparation } 55, \\
\text { after } 4 \text { from } 3 \text { sites in } 25 \text { participants } \\
\text { Postoperative infection } \\
\text { No participant allocated to either } \\
\text { iodine-alcohol or chlorhexidine } \\
\text { developed a post-operative } \\
\text { infection }\end{array}$ & $\begin{array}{l}\text { In addition to the skin preparation on the } \\
\text { surgical site (the foot), the participant's } \\
\text { opposite foot (i.e., the foot not being operated } \\
\text { upon) was scrubbed with a bristle brush for } \\
\text { 3-min before the same preparation was } \\
\text { applied. } \\
\text { For the purposes of analysis, only data from } \\
\text { the surgical site included. } \\
\text { Sample size estimate provided } \\
\text { Follow-up: not defined }\end{array}$ \\
\hline $\begin{array}{l}\text { Kesani } \\
2019 \\
{[20]}\end{array}$ & $\begin{array}{l}\text { Randomised controlled trial } \\
\text { Single centre study }\end{array}$ & Caesarean section & $\begin{array}{l}\text { Group A } \\
\text { povidone iodine-alcohol } \\
\text { (10\% povidone-iodine and then } \\
\text { with surgical spirit) } \\
(n \text { total = 287; included in trial } \\
\text { analysis } n=287) \\
\text { Group B } \\
\text { chlorhexidine alcohol } \\
(2 \% \text { chlorhexidine gluconate } \\
\text { and } 70 \% \text { isopropyl alcohol) } \\
(n \text { total }=273 \text {; included in trial } \\
\text { analysis } n=273)\end{array}$ & $\begin{array}{l}\text { SSI defined as per the CDC } \\
\text { definitions. Superficial and } \\
\text { deep SSI reported. }\end{array}$ & $\begin{array}{l}\text { SSI } \\
\text { All SSI } \\
\text { Iodophor-alcohol 41/287 (14.3\%) } \\
\text { Chlorhexidine-alcohol 19/273 (7.0\%) } \\
\text { Superficial SSI } \\
\text { Iodophor-alcohol 29/287 (10.1\%) } \\
\text { Chlorhexidine-alcohol 15/273 (5.5\%) } \\
\text { Deep SSI } \\
\text { Iodophor-alcohol 12/287 (4.2\%) } \\
\text { Chlorhexidine-alcohol 4/273 (1.5\%) } \\
\text { No difference in microorganisms } \\
\text { from cultured reported. }\end{array}$ & $\begin{array}{l}\text { Sample size estimate not provided } \\
\text { Follow-up: } 30 \text { days postoperatively }\end{array}$ \\
\hline $\begin{array}{l}\text { Ngai } \\
2015 \\
{[21]}\end{array}$ & $\begin{array}{l}\text { Randomised controlled trial } \\
\text { Three-arm study } \\
\text { Two centres }\end{array}$ & Caesarean section & $\begin{array}{l}\text { Group A } \\
\text { povidone iodine-alcohol } \\
\text { (concentrations not stated) } \\
\text { ( } n \text { total }=463 \text {; included in trial } \\
\text { analysis } n=463 \text { ) } \\
\text { Group B } \\
\text { chlorhexidine alcohol } \\
\text { (concentrations not stated) } \\
(n \text { total }=474 \text {; included in trial } \\
\text { analysis } n=474) \\
\text { Group C } \\
\text { combination of povidone } \\
\text { iodine-alcohol (concentrations } \\
\text { not stated) applied first } \\
\text { followed by chlorhexidine } \\
\text { alcohol (concentrations not } \\
\text { stated) } \\
\text { ( } n \text { total }=467 \text {; included in trial } \\
\text { analysis } n=467)\end{array}$ & $\begin{array}{l}\text { Surgical site infection defined } \\
\text { according to the CDC } \\
\text { definition assessed at 2- and } \\
\text { 6-weeks post-cesarean }\end{array}$ & $\begin{array}{l}\text { SSI } \\
\text { All SSI } \\
\text { Iodophor-alcohol 21/463 (4.5\%) } \\
\text { Chlorhexidine-alcohol 21/474 (4.4\%) } \\
\text { Combination 18/467 (3.9\%) } \\
\text { Superficial SSI } \\
\text { Iodophor-alcohol 16/463 (3.5\%) } \\
\text { Chlorhexidine-alcohol 15/474 (3.2\%) } \\
\text { Combination 15/467 (3.2\%) } \\
\text { Deep SSI } \\
\text { Iodophor-alcohol 3/463 (0.6\%) } \\
\text { Chlorhexidine-alcohol 3/474 (0.4\%) } \\
\text { Combination 1/467 (0.2\%) } \\
\text { Organ space } \\
\text { Iodophor-alcohol 2/463 (0.4\%) } \\
\text { Chlorhexidine-alcohol 3/474 (0.6\%) } \\
\text { Combination 2/467 (0.4\%) }\end{array}$ & $\begin{array}{l}\text { Analysis included data for participants } \\
\text { administered povidone iodine-alcohol or } \\
\text { chlorhexidine alcohol arms. } \\
\text { Data from the combination arm with } \\
\text { povidone iodine-alcohol followed by } \\
\text { chlorhexidine alcohol excluded from analysis } \\
\text { Sample size estimate provided } \\
\text { Follow-up: } 30 \text { days postoperatively }\end{array}$ \\
\hline
\end{tabular}


Table 1. Cont.

\begin{tabular}{|c|c|c|c|c|c|c|}
\hline Study & Methods & Surgery Type & Interventions & Outcomes & Results & Notes \\
\hline $\begin{array}{l}\text { Ostrander } \\
2005 \\
{[22]}\end{array}$ & $\begin{array}{l}\text { Randomised controlled trial } \\
\text { Three-arm study } \\
\text { Single centre study }\end{array}$ & Foot and ankle surgery & $\begin{array}{l}\text { Group A } \\
\text { "DuraPrep" [0.7\% available } \\
\text { iodine } / 74 \% \text { isopropyl alcohol } \\
(w / w)] \\
(n \text { total }=40 ; \text { included in trial } \\
\text { analysis } n=40) \\
\\
\text { Group B } \\
\text { "Techni-Care" } \\
{[3.0 \% \text { chloroxylenol] }} \\
(n \text { total = 40; included in trial } \\
\text { analysis } n=40) \\
\text { Group C } \\
\text { "ChloraPrep" } \\
{[2 \% \text { chlorhexidine gluconate }} \\
(w / v) \text { and } 70 \% \text { isopropyl } \\
\text { alcohol }(v / v)] \text {. } \\
(n \text { total }=40 ; \text { included in trial } \\
\text { analysis } n=40)\end{array}$ & $\begin{array}{l}\text { Number of bacterial colonies } \\
\text { forming units pre- and } \\
\text { post-treatment with surgical } \\
\text { site skin preparation: } \\
\text { specimens collected from three } \\
\text { sites: tibia, } 12 \mathrm{~cm} \text { proximal to } \\
\text { the ankle } \\
\text { Joint (labelled as control), } \\
\text { hallucal nail fold (labelled as } \\
\text { hallux), web spaces between } \\
\text { the second and third and } \\
\text { between the fourth and fifth } \\
\text { digits (labelled as toe site). } \\
\text { Postoperative infection rate } \\
\text { was reported as a secondary } \\
\text { outcome. No definition for } \\
\text { infection provided }\end{array}$ & $\begin{array}{l}\text { Positive cultures } \\
\text { Iodophor-alcohol: } 65 \% \text { of hallux } \\
\text { cultures positive, } 45 \% \text { of toe } \\
\text { cultures and } 23 \% \text { of control cultures } \\
\text { positive in } 40 \text { participants } \\
\text { Chlorhexidine-alcohol: } 30 \% \text { of } \\
\text { hallux cultures positive, } 23 \% \text { of toe } \\
\text { cultures and } 10 \% \text { of control cultures } \\
\text { positive in } 40 \text { participants } \\
\text { Chloroxylenol: } 95 \% \text { of hallux } \\
\text { cultures positive, } 98 \% \text { of toe } \\
\text { cultures and } 35 \% \text { of control cultures } \\
\text { positive in } 40 \text { participants } \\
\text { Postoperative infections } \\
\text { Iodophor-alcohol } 0 / 40(0 \%) \\
\text { Chlorhexidine-alcohol } 1 / 40(2.5 \%) \\
\text { Chloroxylenol: } 2 / 40(5.0 \%)\end{array}$ & $\begin{array}{l}\text { All procedures performed by one surgeon } \\
\text { Analysis included data for participants } \\
\text { administered DuraPrep [0.7\% available } \\
\text { iodine } / 74 \% \text { isopropyl alcohol] or ChloraPrep } \\
{[2 \% \text { chlorhexidine gluconate } / 70 \% \text { isopropyl }} \\
\text { alcohol] arms. } \\
\text { Data from the Techni-Care ( } 3.0 \% \\
\text { chloroxylenol) arm excluded from analysis. } \\
\text { Sample size not met } \\
\text { Follow-up: not defined }\end{array}$ \\
\hline $\begin{array}{l}\text { Peel } \\
2019 \\
{[23]}\end{array}$ & $\begin{array}{l}\text { Cluster randomised controlled } \\
\text { trial } \\
\text { Clustered by given day of } \\
\text { surgery } \\
\text { Single centre study }\end{array}$ & $\begin{array}{l}\text { Elective hip or knee } \\
\text { arthroplasty }\end{array}$ & $\begin{array}{l}\text { Group A } \\
1 \% \text { iodine }(w / v) \text { in } 70 \% \text { ethanol } \\
(v / v) \\
(n \text { total }=390 ; \text { included in trial } \\
\text { analysis } n=390) \\
\\
\text { Group B } \\
0.5 \% \text { chlorhexidine gluconate } \\
(w / v) \text { in } 70 \% \text { ethanol }(v / v) \\
(n \text { total }=390 ; \text { included in trial } \\
\text { analysis } n=390)\end{array}$ & $\begin{array}{l}\text { Primary outcome: superficial } \\
\text { wound complication } \\
\text { (composite of superficial } \\
\text { incisional SSI and/ or clinically } \\
\text { significant wound ooze) }\end{array}$ & $\begin{array}{l}\begin{array}{l}\text { Superficial wound complication } \\
\text { (composite of wound ooze and } \\
\text { superficial SSI) } \\
\text { Iodophor-alcohol 15/390 (3.8\%) } \\
\text { Chlorhexidine-alcohol 19/390 (4.9\%) } \\
\text { Wound Ooze } \\
\text { Iodophor-alcohol 13/390 (3.3\%) } \\
\text { Chlorhexidine-alcohol 15/390 (3.8\%) } \\
\text { Adverse reactions } \\
\text { No adverse reactions (skin } \\
\text { irritation/allergy) reported in } \\
\text { either arm }\end{array}\end{array}$ & $\begin{array}{l}\text { Sample size provided } \\
\text { Follow-up: } 30 \text { days for primary outcome and } \\
365 \text { days for secondary outcome } \\
90 \text { Protocol violations observed. } \\
\text { Sensitivity analysis with per-protocol and } \\
\text { as-treated analysis }\end{array}$ \\
\hline
\end{tabular}


Table 1. Cont.

\begin{tabular}{|c|c|c|c|c|c|c|}
\hline Study & Methods & Surgery Type & Interventions & Outcomes & Results & Notes \\
\hline $\begin{array}{l}\text { Rodrigues } \\
2013 \\
{[24]}\end{array}$ & $\begin{array}{l}\text { Randomised controlled trial } \\
\text { Single centre study }\end{array}$ & $\begin{array}{l}\text { Abdominal and thoracic } \\
\text { surgery with subcostal } \\
\text { abdominal, vertical abdominal } \\
\text { and thoracic incision. }\end{array}$ & $\begin{array}{l}\text { Group A } \\
10 \% \text { hydroalcoholic } \\
\text { povidone-iodine } \\
\text { ( } n \text { total = 102; included in trial } \\
\text { analysis } n=102) \\
\\
\text { Group B } \\
0.5 \% \text { alcoholic chlorhexidine } \\
(n \text { total }=103 \text {; included in trial } \\
\text { analysis } n=103)\end{array}$ & $\begin{array}{l}\text { Diagnosis for SSI based on } \\
\text { clinical, microbiological and } \\
\text { radiological findings. } \\
\text { Definition maps to the CDC } \\
\text { definition for SSI. Diagnosis of } \\
\text { SSI required at least one of the } \\
\text { following signs: fever, without } \\
\text { other apparent cause, pain, } \\
\text { heat, swelling, or confluent } \\
\text { erythema around the incision } \\
\text { and extrapolating the } \\
\text { boundaries of the wound, pus } \\
\text { in the incision site or in the } \\
\text { deep soft tissue, or in } \\
\text { organ/cavity handled during } \\
\text { operation; presence of } \\
\text { abscesses or, in the case of deep } \\
\text { tissues, histological or } \\
\text { radiological evidence } \\
\text { suggestive of infection; isolated } \\
\text { microorganism from } \\
\text { theoretically sterile source or } \\
\text { harvested with aseptic } \\
\text { technique from a previously } \\
\text { closed site, and spontaneous } \\
\text { dehiscence of deep tissues. }\end{array}$ & $\begin{array}{l}\text { SSI } \\
\text { All SSI } \\
\text { Iodophor-alcohol 7/102 (6.7\%) } \\
\text { Chlorhexidine-alcohol 11/103 } \\
(10 / 7 \%) \\
\text { Superficial SSI } \\
\text { Iodophor-alcohol 5/102 (4.9\%) } \\
\text { Chlorhexidine-alcohol 9/103 (8.7\%) } \\
\text { Deep SSI } \\
\text { Iodophor-alcohol 1/102 (1.0\%) } \\
\text { Chlorhexidine-alcohol 2/103 (1.9\%) } \\
\text { Organ space } \\
\text { Iodophor-alcohol 1/102 (1.0\%) } \\
\text { Chlorhexidine-alcohol 0/103 (0\%) }\end{array}$ & $\begin{array}{l}\text { Sample size not provided } \\
\text { Follow-up: } 30 \text { days }\end{array}$ \\
\hline $\begin{array}{l}\text { Saltzman } \\
2009 \\
{[25]}\end{array}$ & $\begin{array}{l}\text { Randomised controlled trial } \\
\text { Three-arm study } \\
\text { Single centre study }\end{array}$ & $\begin{array}{l}\text { Shoulder surgery including } \\
\text { shoulder arthroplasty }(n=4)\end{array}$ & $\begin{array}{l}\text { Group A } \\
\text { "DuraPrep" [0.7\% iodophor } \\
\text { and 74\% isopropyl alcohol] } \\
(n \text { total }=50 \text {; included in trial } \\
\text { analysis } n=50) \\
\text { Group B } \\
\text { "ChloraPrep" [2\% } \\
\text { chlorhexidine gluconate and } \\
70 \% \text { isopropyl alcohol] } \\
(n \text { total }=50 \text {; included in trial } \\
\text { analysis } n=50) \\
\text { Group C } \\
\text { povidone-iodine scrub and } \\
\text { paint [0.75\% iodine scrub and } \\
1.0 \% \text { iodine paint }] \\
(n \text { total }=50 \text {; included in trial } \\
\text { analysis } n=50)\end{array}$ & $\begin{array}{l}\text { Rate of positive microbiological } \\
\text { cultures pre- and } \\
\text { post-treatment with surgical } \\
\text { site skin preparation. } \\
\text { Postoperative infection rate } \\
\text { was reported as a secondary } \\
\text { outcome. No definition for } \\
\text { infection provided }\end{array}$ & $\begin{array}{l}\text { Positive cultures } \\
\text { Iodophor-alcohol: } 19 \% \text { of cultures } \\
\text { were positive in } 50 \text { participants } \\
\text { Chlorhexidine-alcohol: } 7 \% \text { of } \\
\text { cultures were positive in } 50 \\
\text { participants } \\
\text { Aqueous-based iodophor: } 31 \% \text { of } \\
\text { cultures were positive in } 50 \\
\text { participants } \\
\text { Postoperative infections } \\
\text { No postoperative infections } \\
\text { developed in any intervention arm }\end{array}$ & $\begin{array}{l}\text { All surgery performed by three surgeons } \\
\text { Analysis included data for participants } \\
\text { administered DuraPrep or ChloraPrep arms. } \\
\text { Data from the povidone-iodine scrub and } \\
\text { paint arm excluded from analysis. } \\
\text { Sample size provided } \\
\text { Follow-up: } 10 \text { months minimum }\end{array}$ \\
\hline
\end{tabular}


Table 1. Cont.

\begin{tabular}{|c|c|c|c|c|c|c|}
\hline Study & Methods & Surgery Type & Interventions & Outcomes & Results & Notes \\
\hline $\begin{array}{l}\text { Savage } \\
2012 \\
{[26]}\end{array}$ & $\begin{array}{l}\text { Randomised controlled trial } \\
\text { Single centre study }\end{array}$ & $\begin{array}{l}\text { Lumbar spine surgery, } \\
\text { including: microdisectomy, } \\
\text { posterior spinal fusion with or } \\
\text { without an associated } \\
\text { interbody fusion } \\
\text { decompression, kyphoplasty }\end{array}$ & $\begin{array}{l}\text { Group A } \\
\text { "DuraPrep" [0.7\% iodophor } \\
\text { and 74\% isopropyl alcohol] } \\
(n \text { total }=50 \text {; included in trial } \\
\text { analysis } n=50) \\
\text { Group B } \\
\text { "ChloraPrep" [2\% } \\
\text { chlorhexidine gluconate and } \\
70 \% \text { isopropyl alcohol] } \\
(n \text { total }=50 \text {; included in trial } \\
\text { analysis } n=50)\end{array}$ & $\begin{array}{l}\text { Rate of positive microbiological } \\
\text { cultures pre- and } \\
\text { post-treatment with surgical } \\
\text { site skin preparation. } \\
\text { Postoperative infection rate } \\
\text { was reported as a secondary } \\
\text { outcome. No definition for } \\
\text { infection provided }\end{array}$ & $\begin{array}{l}\text { Positive cultures } \\
\text { Iodophor-alcohol: } 80 \% \text { of cultures } \\
\text { positive pre-preparation, } 6 \% \\
\text { positive post-preparation, } 32 \% \\
\text { positive post-closure in } 50 \\
\text { participants } \\
\text { Chlorhexidine-alcohol: } 84 \% \text { of } \\
\text { cultures positive pre-preparation, } \\
0 \% \text { positive post-preparation, } 34 \% \\
\text { positive post-closure in } 50 \\
\text { participants } \\
\text { Postoperative infection } \\
\text { Iodophor-alcohol } 0 / 50(0 \%) \\
\text { Chlorhexidine-alcohol } 1 / 50(2.0 \%): \\
\text { All classed as superficial }\end{array}$ & $\begin{array}{l}\text { All surgery performed by four surgeons } \\
\text { Sample size provided } \\
\text { Follow-up: } 6 \text { months minimum }\end{array}$ \\
\hline $\begin{array}{l}\text { Tuuli } \\
2016 \\
{[27]}\end{array}$ & $\begin{array}{l}\text { Randomised controlled trial } \\
\text { Single centre study }\end{array}$ & Caesarean section & $\begin{array}{l}\text { Group A } \\
\text { chlorhexidine-alcohol } \\
\text { combination }[2 \% \text { chlorhexidine } \\
\text { gluconate with } 70 \% \text { isopropyl } \\
\text { alcohol] } \\
\text { ( } n \text { total }=575 \text {; included in trial } \\
\text { analysis } n=575) \\
\text { Group B } \\
\text { iodine-alcohol combination } \\
{[8.3 \% \text { povidone-iodine with }} \\
72.5 \% \text { isopropyl alcohol] } \\
(n \text { total }=572 \text {; included in trial } \\
\text { analysis } n=572)\end{array}$ & $\begin{array}{l}\text { Superficial or deep SSI based } \\
\text { on the CDC/National } \\
\text { Healthcare Safety Network } \\
\text { definitions }\end{array}$ & $\begin{array}{l}\text { SSI } \\
\text { All SSI } \\
\text { Iodophor-alcohol 42/575 (7.3\%) } \\
\text { Chlorhexidine-alcohol 23/572 (4.0\%) } \\
\text { Superficial SSI } \\
\text { Iodophor-alcohol 28/575 (4.9\%) } \\
\text { Chlorhexidine-alcohol 17/572 (3.0\%) } \\
\text { Deep SSI } \\
\text { Iodophor-alcohol 14/575 (2.4\%) } \\
\text { Chlorhexidine-alcohol 6/572 (1.0\%) } \\
\text { Adverse reactions } \\
\text { Iodophor-alcohol 4/575 (0.7\%) } \\
\text { Chlorhexidine-alcohol 2/572 (0.3\%) } \\
\text { In a post hoc analysis, the use of } \\
\text { healthcare resources did not differ } \\
\text { intervention arms }\end{array}$ & $\begin{array}{l}\text { Sample size provided } \\
\text { Follow-up: } 30 \text { days }\end{array}$ \\
\hline
\end{tabular}


Table 1. Cont.

\begin{tabular}{|c|c|c|c|c|c|c|}
\hline Study & Methods & Surgery Type & Interventions & Outcomes & Results & Notes \\
\hline $\begin{array}{l}\text { Veiga } \\
2008 \\
{[29]}\end{array}$ & $\begin{array}{l}\text { Randomised controlled trial } \\
\text { Single centre study }\end{array}$ & $\begin{array}{l}\text { Elective and clean plastic } \\
\text { surgery procedures including: } \\
\text { breast surgery, abdominoplasty, } \\
\text { scar revision, zetaplasty, lipoma } \\
\text { excision }\end{array}$ & $\begin{array}{l}\text { Group A } \\
\text { alcohol solution of povidone- } \\
\text { iodine } 10 \% \\
(n \text { total = 125; included in trial } \\
\text { analysis } n=125) \\
\\
\text { Group B } \\
\text { alcohol solution of } \\
\text { chlorhexidine } 0.5 \% \\
(n \text { total }=125 ; \text { included in trial } \\
\text { analysis } n=125)\end{array}$ & $\begin{array}{l}\text { Primary outcome was } \\
\text { quantitative skin cultures } \\
\text { before and after surgical site } \\
\text { skin preparation. } \\
\text { Surgical site infection was a } \\
\text { secondary outcome, defined } \\
\text { according to the CDC definition }\end{array}$ & $\begin{array}{l}\text { Colony-forming units (standard } \\
\text { deviation) } \\
\text { Iodophor-alcohol: 75.4 (115.9) } \\
\text { colony-forming units } \\
\text { pre-preparation, } 1.3 \text { (5.7) } \\
\text { colony-forming units 2-min after } \\
\text { preparation, 17.6 (64.7) } \\
\text { colony-forming units at end of } \\
\text { surgery in } 125 \text { participants } \\
\text { Chlorhexidine-alcohol: } 93.8 \text { (127.3) } \\
\text { colony-forming units } \\
\text { pre-preparation, 0.3 }(1.3) \\
\text { colony-forming units 2-min after } \\
\text { preparation, } 7.8 \text { (46.1) } \\
\text { colony-forming units at end of } \\
\text { surgery in } 125 \text { participants } \\
\text { SSI } \\
\text { Postoperative infection } \\
\text { Iodophor-alcohol } 4 / 125(03.2 \%) \\
\text { Chlorhexidine-alcohol } 0 / 125(0 \%) \\
\text { All classed as superficial }\end{array}$ & $\begin{array}{l}\text { Study authors reported four infections (in } \\
125 \text { participants) in the iodophor-alcohol } \\
\text { intervention arm calculated as } 3.2 \% \text {. The } \\
\text { study authors however reported this } \\
\text { proportion as " } 1.6 \% \text { " (equating to } 2 \text { infections } \\
\text { in } 125 \text { participants). Given this discrepancy, } \\
\text { for the analysis, the number of events was } \\
\text { transcribed as } 4 \text { infections. } \\
\text { Sample size not provided } \\
\text { Follow-up: } 30 \text { days }\end{array}$ \\
\hline $\begin{array}{l}\mathrm{Xu} \\
2017 \\
{[28]}\end{array}$ & $\begin{array}{l}\text { Randomised controlled trial } \\
\text { Three arm trial } \\
\text { Single centre study }\end{array}$ & $\begin{array}{l}\text { Elective clean soft tissue hand } \\
\text { surgery (e.g., carpal tunnel } \\
\text { release, trigger finger, de } \\
\text { Quervain release, mass excision } \\
\text { or excision ganglion cyst) }\end{array}$ & $\begin{array}{l}\text { Group A } \\
\text { "DuraPrep" [0.7\% iodophor } \\
\text { and 74\% isopropyl alcohol] } \\
\text { ( } n \text { total = } 81 \text {; included in trial } \\
\text { analysis } n=81) \\
\\
\text { Group B } \\
\text { "ChloraPrep" [2\% } \\
\text { chlorhexidine gluconate and } \\
70 \% \text { isopropyl alcohol]. } \\
(n \text { total = 79; included in trial } \\
\text { analysis } n=79) \\
\text { Group C } \\
\text { "Betadine solution" }[10 \% \\
\text { povidone-iodine] } \\
(n \text { total }=80 ; \text { included in trial } \\
\text { analysis } n=80)\end{array}$ & $\begin{array}{l}\text { Rate of positive microbiological } \\
\text { cultures pre- and } \\
\text { post-treatment with surgical } \\
\text { site skin preparation. } \\
\\
\text { Postoperative infection rate } \\
\text { was reported as a secondary } \\
\text { outcome. Postoperative } \\
\text { infection defined as defined as } \\
\text { need for antibiotics or surgical } \\
\text { intervention. }\end{array}$ & $\begin{array}{l}\text { Positive cultures } \\
\text { Iodophor-alcohol: cultures positive } \\
\text { in } 24 \text { of } 81 \text { participants }(29.6 \%) \\
\text { pre-preparation and } 3 \text { of } 81 \\
\text { participants (3.7\%) post-preparation } \\
\text { Chlorhexidine-alcohol: cultures } \\
\text { positive in } 32 \text { of } 79 \text { participants } \\
(40.5 \%) \text { pre-preparation and } 21 \text { of } 79 \\
\text { participants ( } 26.6 \%) \\
\text { post-preparation } \\
\text { Aqueous-iodophor: cultures } \\
\text { positive in } 35 \text { of } 80 \text { participants } \\
(43.8 \%) \text { pre-preparation and } 1 \text { of } 80 \\
\text { participants }(1.3 \%) \text { post-preparation } \\
\text { SSI } \\
\text { Postoperative infections } \\
\text { Iodophor-alcohol } 1 / 81(1.2 \%) \\
\text { Chlorhexidine-alcohol } 2 / 79(2.5 \%) \\
\text { Aqueous-iodophor } 1 / 80(1.3 \%) \\
\text { All classed as superficial }\end{array}$ & $\begin{array}{l}\text { Number of participants allocated to } \\
\text { intervention arms differs across Figure } 1, \\
\text { Table } 2 \text { and in text. For the purposes of the } \\
\text { analysis, the number of participants has been } \\
\text { transcribed keeping with Figure } 1 \text { (Consort } \\
\text { flow diagram). } \\
\text { Analysis included data for participants } \\
\text { administered DuraPrep or ChloraPrep arms. } \\
\text { Data from the Betadine solution arm } \\
\text { excluded from analysis. } \\
\text { Sample size provided } \\
\text { Follow-up: } 42 \text { days }\end{array}$ \\
\hline
\end{tabular}


Table 2. Summary of Effects Table.

\begin{tabular}{|c|c|c|c|c|c|}
\hline \multirow{2}{*}{ Outcomes } & \multicolumn{2}{|c|}{ Absolute Effect } & \multirow{2}{*}{ Absolute Risk Difference } & \multirow{2}{*}{ Relative Effect * $(95 \% \mathrm{CI})$} & \multirow{2}{*}{ Participants (Studies } \\
\hline & Chlorhexidine-Alcohol & Iodophor-Alcohol & & & \\
\hline \multicolumn{6}{|c|}{ All procedures } \\
\hline All SSI & $216 / 3026(7.1 \%)$ & $271 / 2997(9.0 \%)$ & $\begin{array}{l}\text { There were } 19 \text { more SSIs per } \\
1000 \text { participants with } \\
\text { iodophor-alcohol surgical site } \\
\text { skin preparation compared with } \\
\text { chlorhexidine-alcohol }\end{array}$ & $\begin{array}{c}\text { RR } 0.790 \\
\text { (0.669 to } 0.932)\end{array}$ & $\begin{array}{c}6023 \\
\text { (13 studies) }\end{array}$ \\
\hline Superficial SSI & $105 / 2573(4.1 \%)$ & $132 / 2584(5.1 \%)$ & $\begin{array}{l}\text { There were } 10 \text { more superficial } \\
\text { SSIs per } 1000 \text { participants with } \\
\text { iodophor-alcohol surgical site } \\
\text { skin preparation compared with } \\
\text { chlorhexidine-alcohol }\end{array}$ & $\begin{array}{c}\text { RR } 0.807 \\
\text { (0.632 to } 1.032)\end{array}$ & $\begin{array}{c}5157 \\
\text { (12 studies) }\end{array}$ \\
\hline Deep or Organ Space SSI & $67 / 2573(2.6 \%)$ & $79 / 2584(3.1 \%)$ & $\begin{array}{l}\text { There were } 5 \text { more deep or organ } \\
\text { space SSIs per } 1000 \text { participants } \\
\text { with iodophor-alcohol surgical } \\
\text { site skin preparation compared } \\
\text { with chlorhexidine-alcohol }\end{array}$ & $\begin{array}{c}\text { RR } 0.904 \\
\text { (0.664 to } 1.230)\end{array}$ & $\begin{array}{c}5157 \\
\text { (12 studies) }\end{array}$ \\
\hline Adverse events & $2 / 1354(0.15 \%)$ & $4 / 1361(0.29 \%)$ & $\begin{array}{l}\text { There is } 1 \text { more adverse event per } \\
1000 \text { participants with } \\
\text { iodophor-alcohol surgical site } \\
\text { skin preparation compared with } \\
\text { chlorhexidine-alcohol }\end{array}$ & $\begin{array}{c}\text { RR } 0.603 \\
\text { (0.145 to } 2.517)\end{array}$ & $\begin{array}{c}2715 \\
\text { (3 studies) }\end{array}$ \\
\hline \multicolumn{6}{|c|}{ Bone and Joint Surgery } \\
\hline All SSI & $14 / 555(2.5 \%)$ & $4 / 555(0.7 \%)$ & $\begin{array}{l}\text { There were } 18 \text { more SSIs per } \\
1000 \text { participants with } \\
\text { chlorhexidine-alcohol surgical } \\
\text { site skin preparation compared } \\
\text { with iodophor-alcohol }\end{array}$ & $\begin{array}{c}\text { RR } 2.667 \\
\text { (1.051 to } 6.765)\end{array}$ & $\begin{array}{c}1110 \\
\text { (5 studies) }\end{array}$ \\
\hline Superficial SSI & $7 / 555(1.3 \%)$ & $3 / 555(0.5 \%)$ & $\begin{array}{l}\text { There were } 8 \text { more superficial } \\
\text { SSIs per } 1000 \text { participants with } \\
\text { chlorhexidine-alcohol surgical } \\
\text { site skin preparation compared } \\
\text { with iodophor-alcohol }\end{array}$ & $\begin{array}{c}\text { RR } 1.800 \\
\text { (0.607 to } 5.335)\end{array}$ & $\begin{array}{c}1110 \\
\text { (5 studies) }\end{array}$ \\
\hline
\end{tabular}


Table 2. Cont.

Patients or Population: Patients undergoing surgery

Settings: Hospital

Intervention: Alcohol-based chlorhexidine surgical site skin preparation

Comparison: Alcohol-based iodophor surgical site skin preparation

\begin{tabular}{|c|c|c|c|c|c|}
\hline \multirow{2}{*}{ Outcomes } & \multicolumn{2}{|c|}{ Absolute Effect } & \multirow{2}{*}{ Absolute Risk Difference } & \multirow{2}{*}{ Relative Effect* $(95 \% \mathrm{CI})$} & \multirow{2}{*}{ Participants (Studies) } \\
\hline & Chlorhexidine-Alcohol & Iodophor-Alcohol & & & \\
\hline Deep or Organ Space SSI & $16 / 1319(1.2 \%)$ & $31 / 1325(2.3 \%)$ & $\begin{array}{l}\text { There were } 11 \text { more deep or } \\
\text { organ space SSIs per } \\
1000 \text { participants with } \\
\text { iodophor-alcohol surgical site } \\
\text { skin preparation compared with } \\
\text { chlorhexidine-alcohol }\end{array}$ & $\begin{array}{c}\text { RR } 0.522 \\
\text { (0.287 to } 0.952)\end{array}$ & $\begin{array}{c}2644 \\
\text { (3 studies) }\end{array}$ \\
\hline Adverse events & $2 / 572(0.3 \%)$ & $4 / 575(0.7 \%)$ & $\begin{array}{l}\text { There are } 4 \text { more adverse events } \\
\text { per } 1000 \text { participants with } \\
\text { iodophor-alcohol surgical site } \\
\text { skin preparation compared with } \\
\text { chlorhexidine-alcohol }\end{array}$ & $\begin{array}{c}\text { RR } 0.503 \\
\text { (0.092 to } 2.733)\end{array}$ & $\begin{array}{c}1147 \\
\text { (1 study) }\end{array}$ \\
\hline \multicolumn{6}{|c|}{ General surgery (including colorectal and abdominal) } \\
\hline All SSI & $137 / 948(14.5 \%)$ & $158 / 911(17.3 \%)$ & $\begin{array}{l}\text { There were } 28 \text { more SSIs per } \\
1000 \text { participants with } \\
\text { iodophor-alcohol surgical site } \\
\text { skin preparation compared with } \\
\text { chlorhexidine-alcohol }\end{array}$ & $\begin{array}{c}\text { RR } 0.844 \\
\text { (0.686 to } 1.038)\end{array}$ & $\begin{array}{c}1859 \\
\text { (3 studies) }\end{array}$ \\
\hline Superficial SSI & $49 / 495(9.9 \%)$ & $51 / 498(10.2 \%)$ & $\begin{array}{l}\text { There were } 3 \text { more superficial } \\
\text { SSIs per } 1000 \text { participants with } \\
\text { iodophor-alcohol surgical site } \\
\text { skin preparation compared with } \\
\text { chlorhexidine-alcohol }\end{array}$ & $\begin{array}{c}\text { RR } 0.968 \\
\text { (0.667 to } 1.404)\end{array}$ & $\begin{array}{c}993 \\
\text { (2 studies) }\end{array}$ \\
\hline Deep or Organ Space SSI & $44 / 495(8.9 \%)$ & $46 / 498(9.2 \%)$ & $\begin{array}{l}\text { There were } 3 \text { more deep or organ } \\
\text { space SSIs per } 1000 \text { participants } \\
\text { with iodophor-alcohol surgical } \\
\text { site skin preparation compared } \\
\text { with chlorhexidine-alcohol }\end{array}$ & $\begin{array}{c}\text { RR } 0.965 \\
\text { (0.653 to } 1.427)\end{array}$ & $\begin{array}{c}993 \\
\text { (2 studies) }\end{array}$ \\
\hline
\end{tabular}


Table 2. Cont.

Patients or Population: Patients undergoing surgery

Settings: Hospital

Intervention: Alcohol-based chlorhexidine surgical site skin preparation

Comparison: Alcohol-based iodophor surgical site skin preparation

\begin{tabular}{|c|c|c|c|c|c|}
\hline \multirow{2}{*}{ Outcomes } & \multicolumn{2}{|c|}{ Absolute Effect } & \multirow{2}{*}{ Absolute Risk Difference } & \multirow{2}{*}{ Relative Effect * $(95 \% \mathrm{CI})$} & \multirow{2}{*}{ Participants (Studies) } \\
\hline & Chlorhexidine-Alcohol & Iodophor-Alcohol & & & \\
\hline Adverse events & 0/392 (0.0\%) & $0 / 396(0.0 \%)$ & There were no events reported & $\begin{array}{c}\text { RR } 1.010 \\
\text { (0.020 to } 50.784)\end{array}$ & $\begin{array}{c}788 \\
\text { (1 study) }\end{array}$ \\
\hline All SSI & $2 / 204(1.0 \%)$ & $5 / 206(2.4 \%)$ & $\begin{array}{l}\text { There were } 14 \text { more SSIs per } \\
1000 \text { participants with } \\
\text { iodophor-alcohol surgical site } \\
\text { skin preparation compared with } \\
\text { chlorhexidine-alcohol }\end{array}$ & $\begin{array}{c}\text { RR } 0.460 \\
\text { (0.105 to } 2.024)\end{array}$ & $\begin{array}{c}410 \\
\text { (2 studies) }\end{array}$ \\
\hline Superficial SSI & $2 / 204(1.0 \%)$ & $5 / 206(2.4 \%)$ & $\begin{array}{l}\text { There were } 14 \text { more superficial } \\
\text { SSIs per } 1000 \text { participants with } \\
\text { iodophor-alcohol surgical site } \\
\text { skin preparation compared with } \\
\text { chlorhexidine-alcohol }\end{array}$ & $\begin{array}{c}\text { RR } 0.460 \\
\text { (0.105 to } 2.024)\end{array}$ & $\begin{array}{c}410 \\
\text { (2 studies) }\end{array}$ \\
\hline Deep or Organ Space SSI & $0 / 204(0.0 \%)$ & $0 / 206(0.0 \%)$ & There were no events reported & $\begin{array}{c}\text { RR } 1.012 \\
\text { (0.064 to } 16.072)\end{array}$ & $\begin{array}{c}410 \\
\text { (2 studies) }\end{array}$ \\
\hline Adverse events & Not reported & Not reported & Not reported & Not estimable & No studies \\
\hline
\end{tabular}

95\% CI: 95\% Confidence interval; RR: Risk ratio. ${ }^{*}$ Risk Ratio included continuity correction. 
All thirteen included trials compared alcohol-based chlorhexidine to alcohol-based iodophor preparations. The concentration of the chlorhexidine was $0.5 \%$ in six trials $[17,19,20,23,24,29]$ and $2 \%$ in six trials [18,22,25-28]. In one trial [21], the concentration of the agents was not specified. The iodophor comparators included povidone-iodine (concentration range $1-10 \%$ ) or free iodine (concentration range $0.7-1 \%$ ). The alcohol concentration was documented in eight trials and ranged from $70-74 \%$. In one trial [20], the iodophor and alcohol was applied in a two-step process.

Overall, $3026(50.2 \%)$ participants were randomised to chlorhexidine-alcohol preparations and 2997 (49.8\%) participants to alcohol-iodophor preparations (Table 1). Participant follow-up ranged from 3 days to 365 days. Six of the studies applied the CDC criteria for defining SSI $[18,20,21,23,27,29]$. In three studies, infections were defined based on clinical diagnosis $[17,24,28]$ and in four studies, no definitions for infection were provided $[19,22,25,26]$. Two trials $[19,25]$ did not report any SSIs in either arm.

SSIs were reported in 7.1\% (216/3026) participants in the chlorhexidine-alcohol group compared with $9.0 \%(271 / 2997)$ participants in the iodophor-alcohol group (Table 2). The use of chlorhexidine-alcohol was associated with a reduction in risk of SSI on pooled analysis (RR 0.790; 95\% CI 0.669, 0.932: $\mathrm{I}^{2} 38.33 \%$ (95\% CI 0 to $68.0 \%$ ): and Figure 2). Results were similar when analysed without continuity correction (RR $0.782 ; 95 \%$ CI 0.662 , 0.924: $\mathrm{I}^{2} 38.43 \%(95 \%$ CI 0-70.0)).

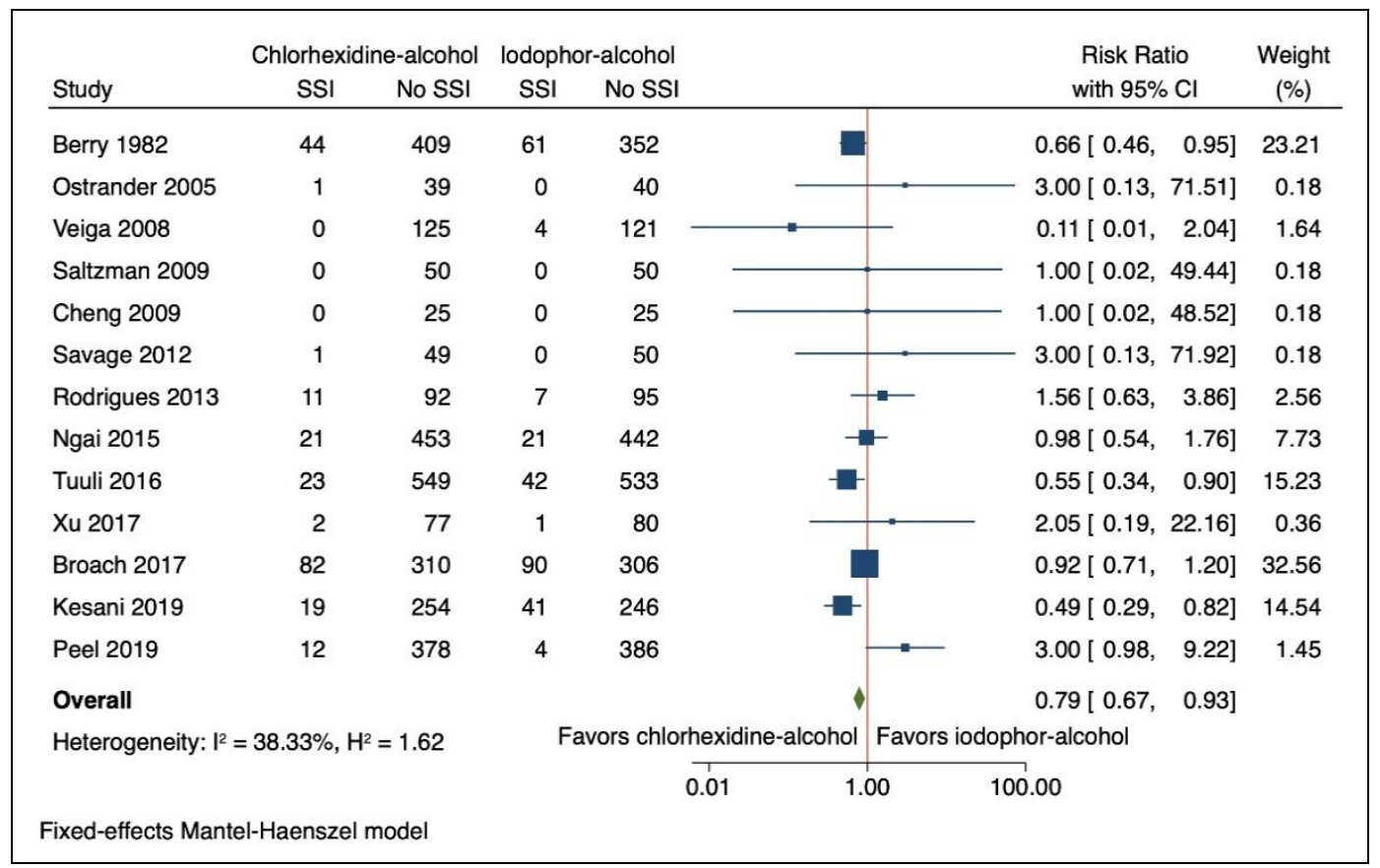

Figure 2. Forest Plot Comparing Risk of Surgical Site Infection (SSI) with Chlorhexidine-Alcohol to Iodophor-Alcohol Surgical Site Skin Preparation. Navy square represents the effect estimates from the individual studies, the size of the square is proportional to the weight of the study. The horizontal line represents the $95 \%$ confidence interval of the study estimate. The green diamond represents the pooled effect size.

On planned sub-group analysis examining different procedure groups (Figure 3), chlorhexidine-alcohol was associated with a reduction in SSIs in caesarean surgery (RR 0.614; $95 \%$ CI $0.453,0.831$ : I $\mathrm{I}^{2} 39.81 \%$ (95\% CI 0 to 81.4$)$ ). In contrast, the use of chlorhexidinealcohol preparation was associated with an increased risk of SSI in bone and joint surgery (RR 2.667; 95\% CI 1.051, 6.765: $\mathrm{I}^{2} 0.00 \%$ (95\% CI 0 to 35.7)). In general surgery and skin and soft tissue surgery, there was no association with skin preparation and risk of SSI (RR 0.844; $95 \%$ CI 0.686, 1.038: I $\mathrm{I}^{2} 49.55 \%$ (95\% CI 0 to 85.4 ) and RR 0.460; 95\% CI 0.105, 2.024: I $\mathrm{I}^{2}$ $58.83 \%$ (95\% CI 0 to 90.2 ), respectively). 


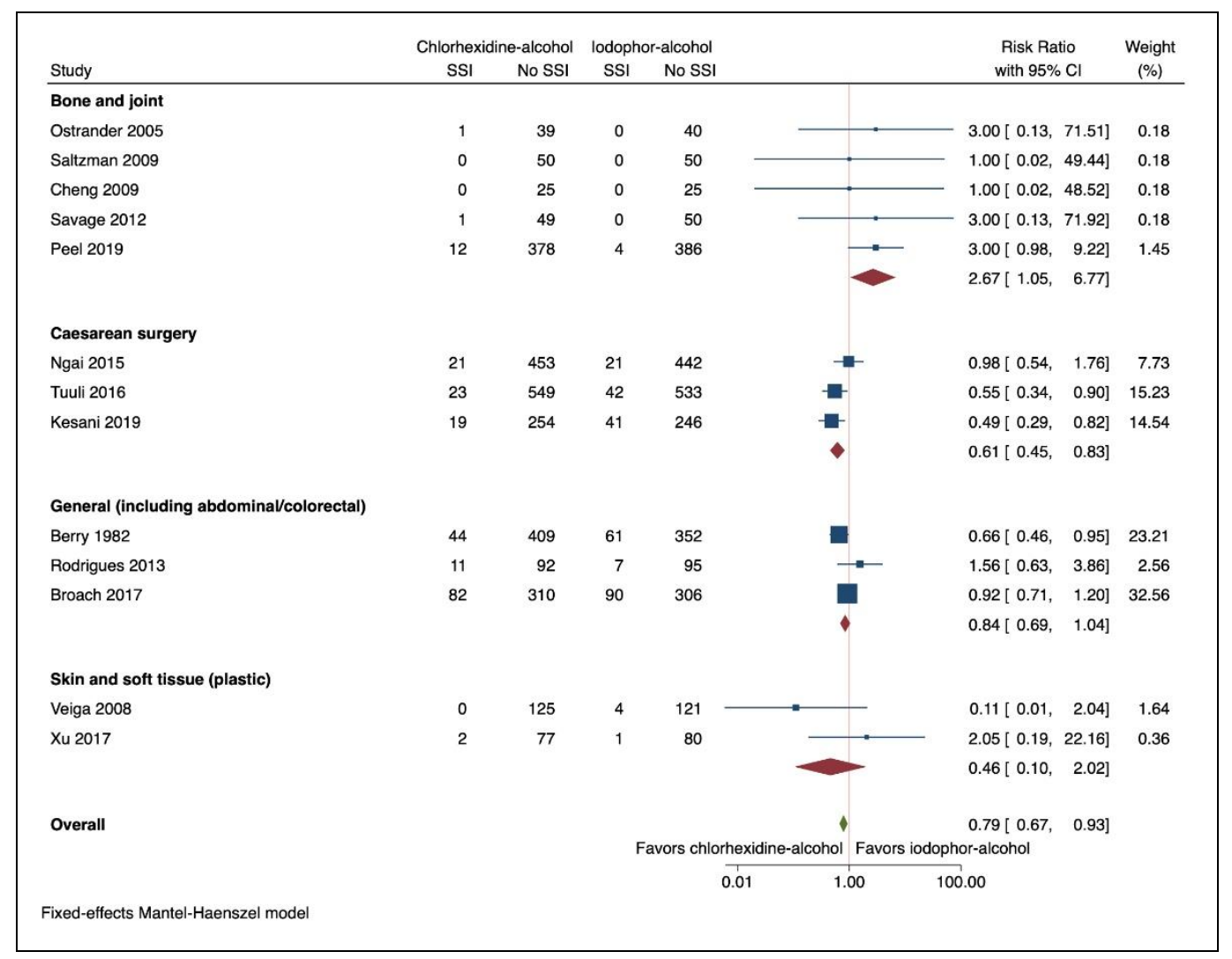

Figure 3. Forest Plot Comparing Risk of Surgical Site Infection (SSI) with Chlorhexidine-Alcohol to Iodophor-Alcohol Surgical Site Skin Preparation According to Procedure Groups. Navy square represents the effect estimates from the individual studies, the size of the square is proportional to the weight of the study. The horizontal line represents the $95 \%$ confidence interval of the study estimate. The red diamond represents the pooled effect size for the specified procedure group. The green diamond represents the pooled effect size.

Repeating the analysis without continuity correction did not alter the findings for caesarean, general or skin and soft tissue surgery (Caesarean Surgery RR 0.614, 95\% CI $0.453,0.831$, General Surgery RR $0.844 ; 95 \%$ CI $0.686,1.038$ and, Skin and Soft Tissue Surgery RR $0.495 ; 95 \%$ CI 0.097, 2.517). While still at increased risk, using chlorhexidine-alcohol preparation for bone and joint surgery was no longer statistically significant (RR 3.000; 95\% CI 0.995, 9.045).

Examining the impact of alcohol-based surgical site skin preparation according to SSI classification did not reveal any significant associations between different alcohol-based surgical skin site preparation agents for superficial SSI ( $n=12$ trials, RR $0.807 ; 95 \%$ CI 0.632 , 1.032: $\mathrm{I}^{2}=0.00 \%(95 \%$ CI 0 to 71.0)) or deep and organ/space SSI ( $n=12$ trials, RR 0.904; 95\% CI 0.664, 1.230: $\mathrm{I}^{2}=0.00 \%(95 \%$ CI 0 to 54.9)) (Table 2).

Adverse events, including allergic reactions to the preparation, were reported in three studies [18,23,27] (Tables 1 and 2). Adverse reactions were rare, occurring in $0.15 \%$ of participants allocated to chlorhexidine-alcohol $(2 / 1354)$ and $0.29 \%$ allocated to iodophoralcohol (4/1361, $p=0.687)$. Tuuli et al. [27] reported on healthcare resource utilisation including emergency room visits and did not find any difference between chlorhexidinealcohol and iodine-alcohol. No other study reported on health economic outcomes.

The risk of bias was assessed as low in two studies [23,27] (Figure 4A). In nine trials, the risk of bias was unclear, particularly the risk of performance and detection bias $[17-21,25,26,28,29]$. There was high risk of selection bias determined in the trials by Rodrigues et al. [24] and Ostrander et al. [22] (Figure 4A). Overall, the risk of reporting bias and attrition bias was low (Figure 4B). Excluding the trials with high risk of bias did not alter the overall estimates $\left(n=11\right.$ trials, RR 0.765; 95\% CI 0.646, 0.907; $p=0.0020: \mathrm{I}^{2}=39.72 \%$ 
(95\% CI 0 to 70.3$)$ ), however, on sub-group analysis of different procedure groups, when the trial by Ostrander et al. was excluded, chlorhexidine-alcohol preparation for bone and joint surgery was no longer statistically significant (RR 2.636; $95 \%$ CI $0.995,6.983 ; \mathrm{I}^{2}=0.00 \%$ (95\% CI 0 to 66.1$)$ ).

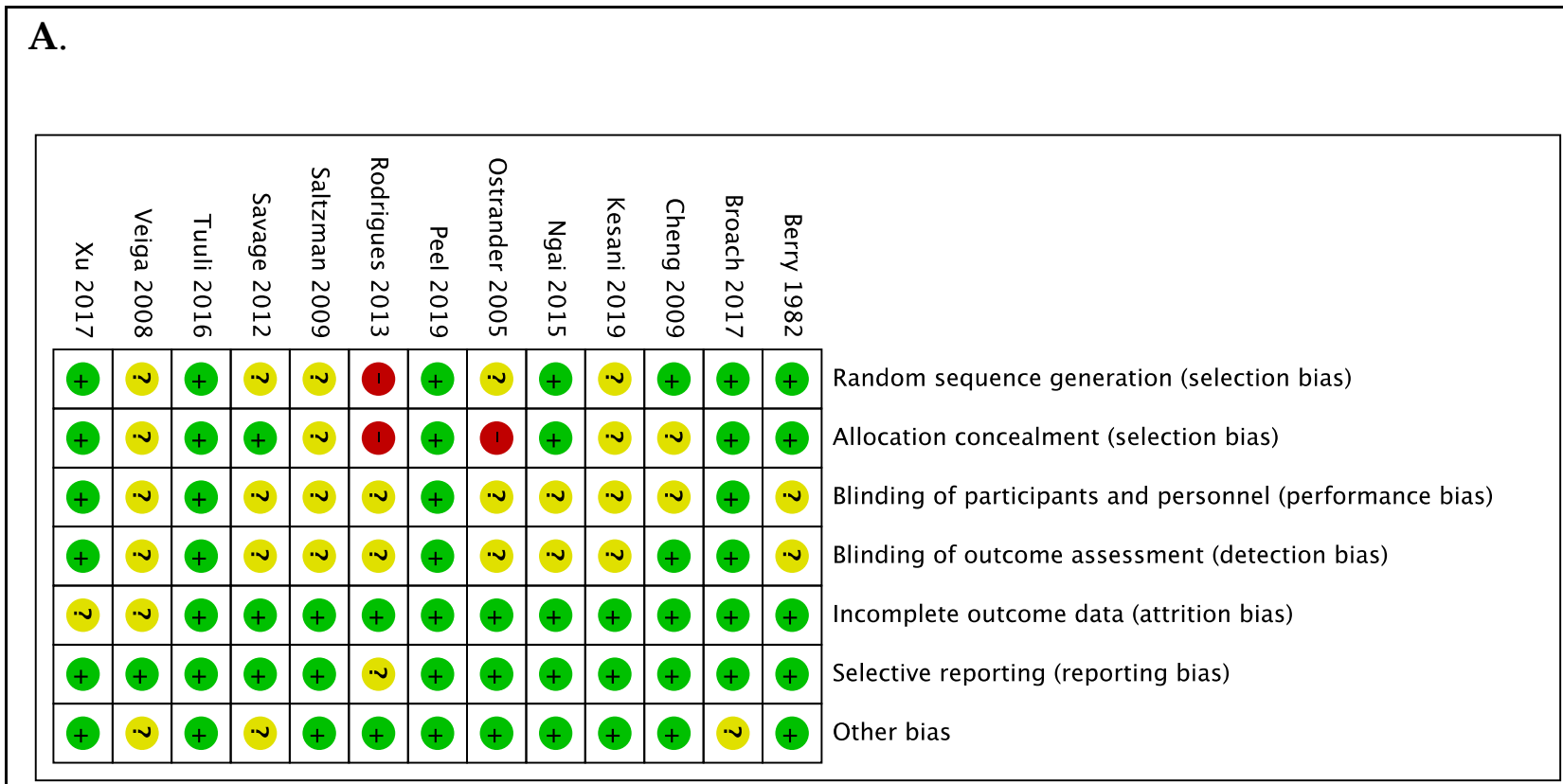

B.

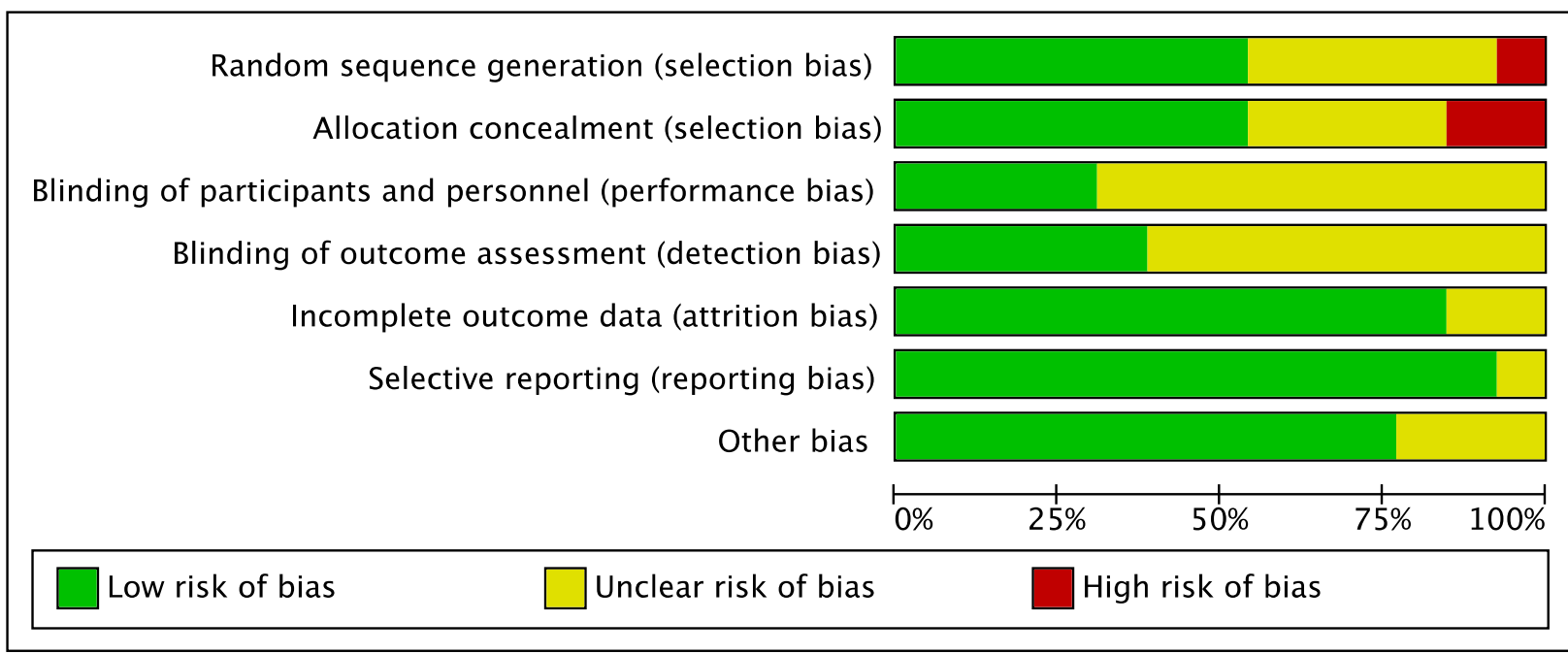

Figure 4. (A). Summary of risk of bias for each trial: low (+), high (-), or unclear (?) and (B). Each risk of bias item presented as percentages across all included studies.

The funnel plot (Figure 5A) was asymmetric. When repeated according to procedure group, the plots were symmetric (Figure $5 \mathrm{~B}$ ) although the number of the trials was small, thereby limiting analysis for bias. 


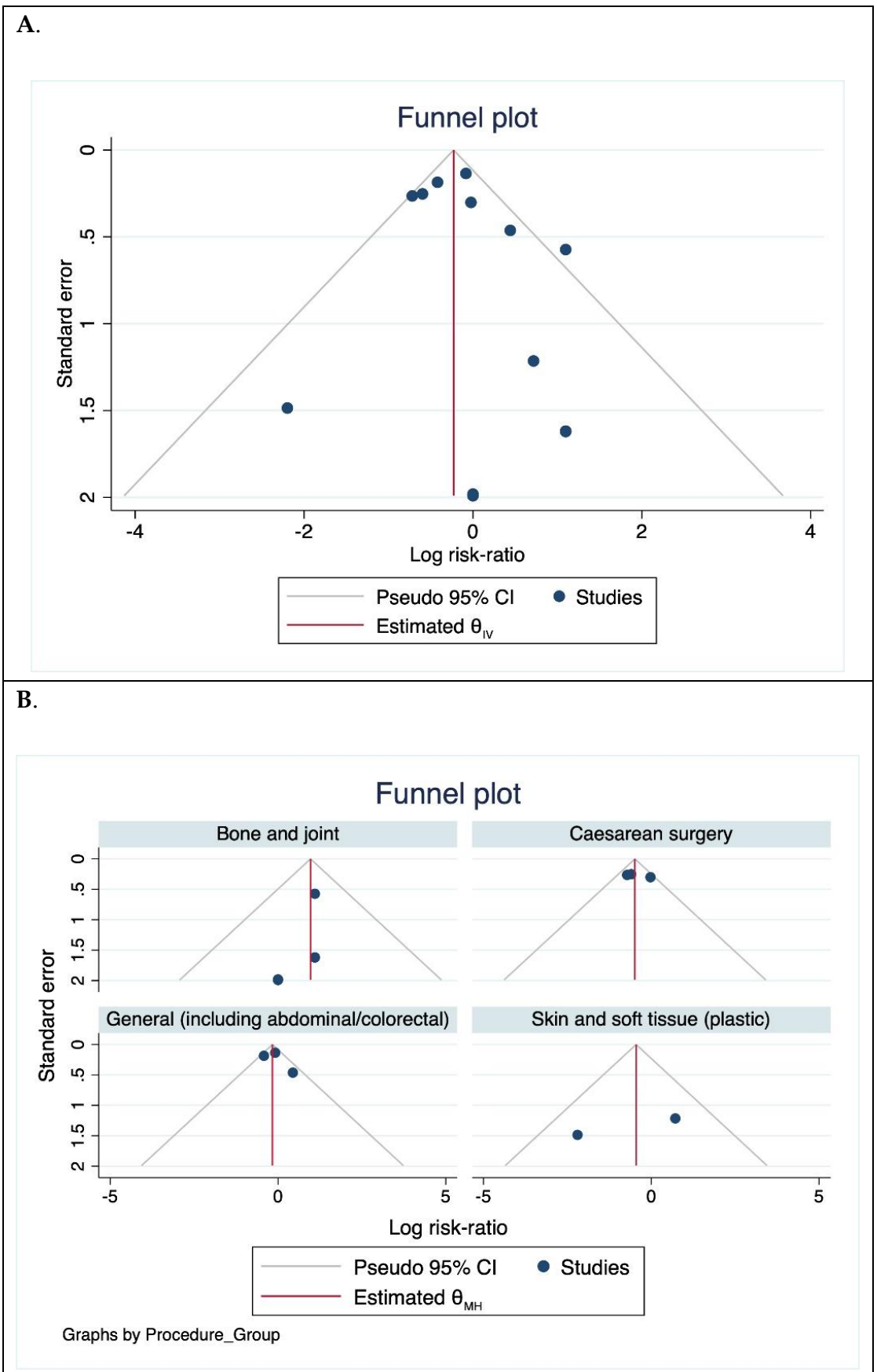

Figure 5. Funnel plot assessing publication bias. (A). For all trials included and (B). According to procedure groups.

\section{Discussion}

Overall, our study, including 6023 participants, showed the use of chlorhexidinealcohol skin preparations was associated with a $21 \%$ reduction in the relative risk of SSI compared to iodophor-alcohol agents. This equates to an absolute difference of 19 fewer infections per 1000 patients undergoing surgery. A key finding however, suggests that the efficacy of alcohol-based surgical site skin preparation agents may differ according to the surgical procedure group. In caesarean surgery, the use of chlorhexidine-alcohol preparations was associated with a 39\% reduction in the relative risk of SSIs. In contrast, chlorhexidine-alcohol skin preparation agents were associated with a 2.7-fold increased risk of SSIs in bone and joint surgery. In the other procedure groups, general surgery and 
skin and soft tissue surgery, there was no demonstrated difference between the alcoholbased agents.

The observation in the bone and joint surgery cohort differs from the WHO guidelines which recommended that alcohol-based chlorhexidine solutions should be used. The metaanalysis performed by the WHO Guideline writing group include three studies examining bone and joint surgery $[19,25,26]$. The estimates in bone and joint surgery were influenced by one large study by Peel et al. [23] although examination of the forest plot suggests the majority of trials in this surgical group favoured alcohol-based iodophor skin preparation agents. The larger study by Peel et al. $(n=780)$ [23] examined skin preparation agents in arthroplasty surgery and was reported after the WHO guidelines were published.

The findings in caesarean surgery were influenced by the trials by Tuuli et al. [27] and Kesani et al. [20]. Of note, the trial by Tuuli et al. was published outside systematic review time limits for the WHO meta-analysis however, was "exceptionally included" [3,7]. This trial also was published after the specified time limits for the CDC meta-analysis [8] and was not included in the meta-analysis, potentially accounting for the differing findings between the two guidelines.

The differences observed, particularly between bone and joint surgery and caesarean surgery, may be due to differences in the populations, particularly age and gender. It may also reflect the differences in infection control approaches, for example, screening and decolonisation for Staphylococcus aureus is a recommended strategy in orthopaedic surgery whereas the role has not been established in caesarean surgery $[7,8]$. The causative organisms of SSI also differ between these surgical groups: Staphylococcus species are the most common bacteria isolated in bone and joint infections, compared with Gram negative bacteria and anaerobes in caesarean surgery $[30,31]$. The majority of the cohort in the bone and joint surgery group underwent procedures involving the implantation of prosthetic material. Infections involving prosthetic material differ from other types of SSIs due to the propensity of organisms involved in medical device infections to form biofilm [8]. This observation raises the possibility that the differences between bone and joint and caesarean surgery may relate to different anti-biofilm properties of the skin preparation agents. Both chlorhexidine and iodophors display anti-biofilm properties [32-34]. The effectiveness of the agents forming biofilms may be concentration dependent [34,35]. In a trial by Smith and colleagues, lower concentrations of chlorhexidine were less effective at eradicating methicillin resistant Staphylococcus aureus biofilm compared to 2 and $4 \%$ concentrations [35]. The trial by Peel et al. used $0.5 \%$ chlorhexidine however, conversely there were more cases of Staphylococcus aureus SSI in the iodophor-alcohol arm (3/4 SSIs: 75\%) compared with the chlorhexidine-alcohol arm (3/12 SSIs: 25\%) [23].

Given that this meta-analysis included a small number of trials $(n=13)$, the observed difference may be a false positive finding, due to chance. Particularly for the analysis according to procedure group, the estimates must be interpreted with caution given the small number of trials included in each sub-group.

The trials included in this study compared different concentrations and formulations of skin preparation agents. The impact on the estimates of differing concentrations is unclear. There is limited data on the optimal concentration of agents, with no head-to-head trials comparing concentrations $[3,7,9,36]$. In addition, a range of definitions for SSI were applied in the included studies and participant follow-up differed. The heterogeneity, however, was low. Finally, the majority of trials included had unclear risk of bias, particularly for performance and detection biases.

\section{Conclusions}

The results of this meta-analysis suggest that the use of alcohol-based chlorhexidine surgical site skin preparation for caesarean section is associated with a lower risk of surgical site infections compared with alcohol-based iodophors. The opposite finding was observed for bone and joint surgical procedures, raising the possibility that the optimal skin preparation agent may differ with surgical procedures. However, when excluding studies 
at risk of major bias, this difference for bone and joint procedures was no longer significant. These observations must be interpreted with caution and require further investigation to corroborate these findings and to determine if there is a biological mechanism(s) explaining these findings. Further, larger trials, particularly in other surgical procedure groups, are warranted.

Author Contributions: Conceptualization, T.N.P. and E.W.; methodology, all authors; formal analysis, all authors; data curation, T.N.P. and E.W.; writing —original draft preparation, T.N.P.; writingreview and editing, all. All authors have read and agreed to the published version of the manuscript.

Funding: Associate Professor Trisha Peel is supported by a National Health and Medical Research Council (NHMRC) of Australia Career Development Fellowship (APP1140350). The funder (NHMRC) had no role in the design, data analysis or the preparation of the manuscript.

Institutional Review Board Statement: Not applicable.

Informed Consent Statement: Not applicable.

Data Availability Statement: Not applicable.

Conflicts of Interest: The authors declare no conflict of interest.

\section{Appendix A. Search Strategy \\ Ovid MEDLINE search terms.}

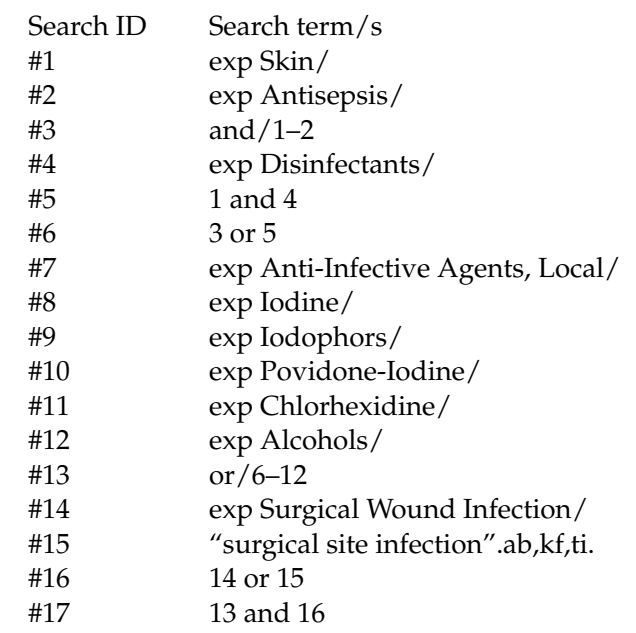

\section{OVID EMBASE and CINAHL search terms.}

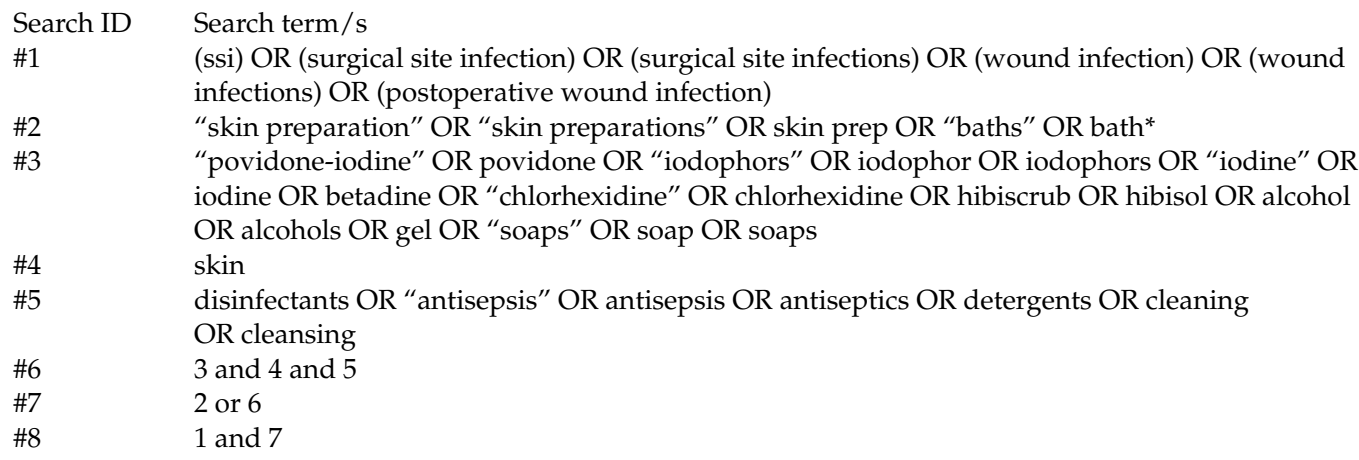




\section{Cochrane Library search terms.}

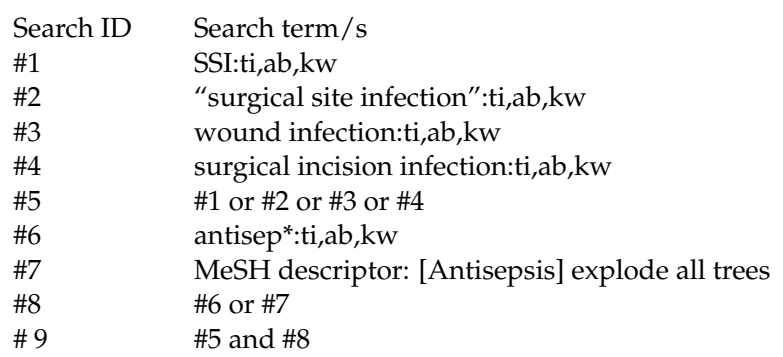

\section{PubMed}

((("surgical wound infection"[Mesh] OR surgical site infection* [TIAB] OR "SSI" OR "SSIs" OR surgical wound infection* [TIAB] OR surgical infection*[TIAB] OR post-operative wound infection* [TIAB] OR postoperative wound infection* [TIAB] OR wound infection*[TIAB]) OR (("preoperative care"[Mesh] OR "preoperative care" OR "pre-operative care" OR "perioperative care"[Mesh] OR "perioperative care" OR "peri-operative care" OR perioperative OR intraoperative OR "perioperative period"[Mesh] OR "intraoperative period"[Mesh]) AND ("infection"[Mesh] OR infection [TIAB])) AND ("skin preparation" [TIAB] OR "skin preparations" [TIAB] OR skin prep [TIAB] OR "baths"[Mesh] OR bath*[TIAB] OR (("povidone-iodine"[Mesh] OR povidone OR "iodophors"[Mesh] OR iodophor OR iodophors OR "iodine"[Mesh] OR iodine OR betadine OR "triclosan"[Mesh] OR triclosan OR "chlorhexidine"[Mesh] OR chlorhexidine OR hibiscrub OR hibisol OR alcohol OR alcohols OR Gel OR "soaps"[Mesh] OR soap [TIAB] OR soaps [TIAB]) AND skin AND (disinfectants OR "antisepsis"[Mesh] OR antisepsis OR antiseptics OR detergents OR cleaning OR cleansing))).

\section{References}

1. Weiser, T.G.; Haynes, A.B.; Molina, G.; Lipsitz, S.R.; Esquivel, M.M.; Uribe-Leitz, T.; Fu, R.; Azad, T.; Chao, T.E.; Berry, W.R.; et al. Estimate of the global volume of surgery in 2012: An assessment supporting improved health outcomes. Lancet 2015, 385 (Suppl. S2), S11. [CrossRef]

2. $\quad$ Bratzler, D.W.; Dellinger, E.P.; Olsen, K.M.; Perl, T.M.; Auwaerter, P.G.; Bolon, M.K.; Fish, D.N.; Napolitano, L.M.; Sawyer, R.G.; Slain, D.; et al. Clinical practice guidelines for antimicrobial prophylaxis in surgery. Surg. Infect. 2013, 14, 73-156. [CrossRef] [PubMed]

3. World Health Organization. Global Guidelines for the Prevention of Surgical Site Infection; Contract No.: WHO Guidelines Approved by the Guidelines Review Committee; WHO: Geneva, Switzerland, 2016.

4. Zimlichman, E.; Henderson, D.; Tamir, O.; Franz, C.; Song, P.; Yamin, C.K.; Keohane, C.; Denham, C.R.; Bates, D.W. Health care-associated infections: A meta-analysis of costs and financial impact on the us health care system. JAMA Intern. Med. 2013, 173, 2039-2046. [CrossRef] [PubMed]

5. Mangram, A.J.; Horan, T.C.; Pearson, M.L.; Silver, L.C.; Jarvis, W.R. Guideline for prevention of surgical site infection, 1999. Hospital Infection Control Practices Advisory Committee. Infect. Control Hosp. Epidemiol. 1999, 20, 250-278; quiz 79-80. [CrossRef] [PubMed]

6. Dumville, J.C.; McFarlane, E.; Edwards, P.; Lipp, A.; Holmes, A.; Liu, Z. Preoperative skin antiseptics for preventing surgical wound infections after clean surgery. Cochrane Database Syst. Rev. 2015. [CrossRef]

7. Allegranzi, B.; Bischoff, P.; de Jonge, S.; Kubilay, N.Z.; Zayed, B.; Gomes, S.M.; Abbas, M.; Atema, J.J.; Gans, S.; van Rijen, M.; et al. New WHO recommendations on preoperative measures for surgical site infection prevention: An evidence-based global perspective. Lancet Infect. Dis. 2016, 16, e276-e287. [CrossRef]

8. Berríos-Torres, S.I.; Umscheid, C.A.; Bratzler, D.W.; Leas, B.; Stone, E.C.; Kelz, R.R.; Reinke, C.E.; Morgan, S.; Solomkin, J.S.; Mazuski, J.E.; et al. Centers for Disease Control and Prevention Guideline for the Prevention of Surgical Site Infection, 2017. JAMA Surg. 2017, 152, 784-791. [CrossRef] [PubMed]

9. Maiwald, M.; Chan, E.S. The forgotten role of alcohol: A systematic review and meta-analysis of the clinical efficacy and perceived role of chlorhexidine in skin antisepsis. PLoS ONE 2012, 7, e44277. [CrossRef] [PubMed]

10. McDonnell, G.; Russell, A.D. Antiseptics and disinfectants: Activity, action, and resistance. Clin. Microbiol. Rev. 1999, 12, 147-179. [CrossRef]

11. Moher, D.; Liberati, A.; Tetzlaff, J.; Altman, D.G.; Group, P. Preferred reporting items for systematic reviews and meta-analyses: The PRISMA statement. PLoS Med. 2009, 6, e1000097. [CrossRef]

12. Higgins, J.P.; Altman, D.G.; Gøtzsche, P.C.; Jüni, P.; Moher, D.; Oxman, A.D.; Savović, J.; Schulz, K.F.; Weeks, L.; Sterne, J.A. The Cochrane Collaboration's tool for assessing risk of bias in randomised trials. BMJ 2011, 343, d5928. [CrossRef] 
13. Sterne, J.A.; Sutton, A.J.; Ioannidis, J.P.; Terrin, N.; Jones, D.R.; Lau, J.; Carpenter, J.; Rücker, G.; Harbord, R.M.; Schmid, C.H.; et al. Recommendations for examining and interpreting funnel plot asymmetry in meta-analyses of randomised controlled trials. BMJ 2011, 343, d4002. [CrossRef]

14. Greenland, S.; Robins, J.M. Estimation of a common effect parameter from sparse follow-up data. Biometrics 1985, 41, 55-68. [CrossRef]

15. Mantel, N.; Haenszel, W. Statistical Aspects of the Analysis of Data From Retrospective Studies of Disease. J. Natl. Cancer Inst. 1959, 22, 719-748. [PubMed]

16. Cox, D.R. The continuity correction. Biometrika 1970, 57, 217-219. [CrossRef]

17. Berry, A.R.; Watt, B.; Goldacre, M.J. A comparison of the use of povidone-iodine and chlorhexidine in the prophylaxis of post-operative wound infection. J. Hosp. Infect. 1982, 3, 402. [CrossRef]

18. Broach, R.B.; Paulson, E.C.; Scott, C.; Mahmoud, N.N. Randomized Controlled Trial of Two Alcohol-based Preparations for Surgical Site Antisepsis in Colorectal Surgery. Ann. Surg. 2017, 266, 946-951. [CrossRef] [PubMed]

19. Cheng, K.; Robertson, H.; St Mart, J.P.; Leanord, A.; McLeod, I. Quantitative analysis of bacteria in forefoot surgery: A comparison of skin preparation techniques. Foot Ankle Int. 2009, 30, 992-997. [CrossRef] [PubMed]

20. Kesani, V.; Talasila, S. Chlorhexidine-alcohol versus Povidone-Iodine-alcohol for surgical site antisepsis in caesarean section. Int. J. Reprod. Contracept. Obstet. Gynecol. 2018, 8, 1360. [CrossRef]

21. Ngai, I.; Govindappagari, S.; Van Arsdale, A.; Judge, N.E.; Neto, N.; Bernstein, J.; Garry, D. LB1: Skin preparation in cesarean birth for prevention of surgical site infection (SSI): A prospective randomized clinical trial. Am. J. Obstet. Gynecol. 2015, 212 , S424. [CrossRef]

22. Ostrander, R.V.; Botte, M.J.; Brage, M.E. Efficacy of surgical preparation solutions in foot and ankle surgery. J. Bone Joint Surg. Am. 2005, 87, 980-985. [CrossRef]

23. Peel, T.N.; Dowsey, M.M.; Buising, K.L.; Cheng, A.C.; Choong, P.F.M. Chlorhexidine-alcohol versus iodine-alcohol for surgical site skin preparation in an elective arthroplasty (ACAISA) study: A cluster randomized controlled trial. Clin. Microbiol. Infect. 2019, 25, 1239-1245. [CrossRef]

24. Rodrigues, A.L. Incidence of surgical site infection with pre-operative skin preparation using $10 \%$ polyvidone-iodine and $0.5 \%$ chlorhexidine-alcohol. Rev. Col. Bras. Cir. 2013, 40, 443-448. [CrossRef] [PubMed]

25. Saltzman, M.D.; Nuber, G.W.; Gryzlo, S.M.; Marecek, G.S.; Koh, J.L. Efficacy of surgical preparation solutions in shoulder surgery. J. Bone Joint Surg. Am. 2009, 91, 1949-1953. [CrossRef] [PubMed]

26. Savage, J.W.; Weatherford, B.M.; Sugrue, P.A.; Nolden, M.T.; Liu, J.C.; Song, J.K.; Haak, M.H. Efficacy of surgical preparation solutions in lumbar spine surgery. J. Bone Joint Surg. Am. 2012, 94, 490-494. [CrossRef] [PubMed]

27. Tuuli, M.G.; Liu, J.; Stout, M.J.; Martin, S.; Cahill, A.G.; Odibo, A.O.; Colditz, G.A.; Macones, G.A. A Randomized Trial Comparing Skin Antiseptic Agents at Cesarean Delivery. N. Engl. J. Med. 2016, 374, 647-655. [CrossRef]

28. Xu, P.Z.; Fowler, J.R.; Goitz, R.J. Prospective Randomized Trial Comparing the Efficacy of Surgical Preparation Solutions in Hand Surgery. Hand 2017, 12, 258-264. [CrossRef] [PubMed]

29. Veiga, D.F.; Damasceno, C.A.; Veiga-Filho, J.; Figueiras, R.G.; Vieira, R.B.; Florenzano, F.H.; Juliano, Y.; Ferreira, L.M. Povidone iodine versus chlorhexidine in skin antisepsis before elective plastic surgery procedures: A randomized controlled trial. Plast. Reconstr. Surg. 2008, 122, 170e-171e. [CrossRef] [PubMed]

30. European Centre for Disease Prevention and Control. Healthcare-Associated Infections: Surgical Site Infections; ECDC: Stockholm, Sweden, 2019.

31. Worth, L.J.; Bull, A.L.; Spelman, T.; Brett, J.; Richards, M.J. Diminishing surgical site infections in Australia: Time trends in infection rates, pathogens and antimicrobial resistance using a comprehensive Victorian surveillance program, 2002-2013. Infect. Control Hosp. Epidemiol. 2015, 36, 409-416. [CrossRef] [PubMed]

32. Schwechter, E.M.; Folk, D.; Varshney, A.K.; Fries, B.C.; Kim, S.J.; Hirsh, D.M. Optimal irrigation and debridement of infected joint implants: An in vitro methicillin-resistant Staphylococcus aureus biofilm model. J. Arthroplast. 2011, 26 (Suppl. S6), 109-113. [CrossRef]

33. Staneviciute, E.; Na'amnih, W.; Kavaliauskas, P.; Prakapaite, R.; Ridziauskas, M.; Kevlicius, L.; Kirkliauskiene, A.; Zabulis, V.; Urboniene, J.; Triponis, V. New in vitro model evaluating antiseptics' efficacy in biofilm-associated Staphylococcus aureus prosthetic vascular graft infection. J. Med. Microbiol. 2019, 68, 432-439. [CrossRef] [PubMed]

34. Schmidt, K.; Estes, C.; McLaren, A.; Spangehl, M.J. Chlorhexidine Antiseptic Irrigation Eradicates Staphylococcus epidermidis From Biofilm: An In Vitro Study. CORR 2018, 476, 648-653. [CrossRef] [PubMed]

35. Smith, D.C.; Maiman, R.; Schwechter, E.M.; Kim, S.J.; Hirsh, D.M. Optimal Irrigation and Debridement of Infected Total Joint Implants with Chlorhexidine Gluconate. J. Arthroplast. 2015, 30, 1820-1822. [CrossRef]

36. Maiwald, M.; Widmer, A.F. WHO's recommendation for surgical skin antisepsis is premature. Lancet Infect. Dis. 2017, 17, 1023-1024. [CrossRef] 